\title{
Selective SIRP $\alpha$ blockade reverses tumor T cell exclusion and overcomes cancer immunotherapy resistance
}

\author{
Vanessa Gauttier, ${ }^{1}$ Sabrina Pengam, ${ }^{1}$ Justine Durand, ${ }^{1}$ Kevin Biteau, ${ }^{1}$ Caroline Mary, ${ }^{1}$ Aurore Morello, ${ }^{1}$ Mélanie Néel, ${ }^{2,3,4}$ \\ Georgia Porto, ${ }^{2,3}$ Géraldine Teppaz, ${ }^{1}$ Virginie Thepenier, ${ }^{1}$ Richard Danger, ${ }^{2,3,4}$ Nicolas Vince, ${ }^{2,3}$ Emmanuelle Wilhelm, ${ }^{1}$ \\ Isabelle Girault, ${ }^{1}$ Riad Abes, ${ }^{1}$ Catherine Ruiz, ${ }^{1}$ Charlène Trilleaud, ${ }^{1,2,3}$ Kerry Ralph, ${ }^{5}$ E. Sergio Trombetta, ${ }^{5}$ \\ Alexandra Garcia, ${ }^{2,3,4}$ Virginie Vignard, ${ }^{4,6}$ Bernard Martinet, ${ }^{2,3}$ Alexandre Glémain, ${ }^{2,3}$ Sarah Bruneau, ${ }^{2,3}$ Fabienne Haspot, ${ }^{2,3}$ \\ Safa Dehmani,, 1,2,3 Pierre Duplouye,, ${ }^{2,3}$ Masayuki Miyasaka, ${ }^{7}$ Nathalie Labarrière, ${ }^{6}$ David Laplaud, ${ }^{2,3,4}$ Stéphanie Le Bas-Bernardet, ${ }^{2,3}$ \\ Christophe Blanquart, ${ }^{6}$ Véronique Catros, ${ }^{8}$ Pierre-Antoine Gouraud, ${ }^{2,3}$ Isabelle Archambeaud, ${ }^{4,9}$ Hélène Aublé, ${ }^{4,9,10}$ \\ Sylvie Metairie, ${ }^{4,9}$ Jean-François Mosnier, ${ }^{2,3,11}$ Dominique Costantini, ${ }^{1}$ Gilles Blancho, ${ }^{2,3,4}$ Sophie Conchon, ${ }^{2,3}$ \\ Bernard Vanhove, ${ }^{1}$ and Nicolas Poirier ${ }^{1}$
}

'OSE Immunotherapeutics, Nantes, France. ${ }^{2}$ Université de Nantes, INSERM, Centre de Recherche en Transplantation et Immunologie, UMR 1064, ${ }^{3}$ Institut de Transplantation Urologie Néphrologie (ITUN), F-44000 Nantes, France. ${ }^{4}$ CHU Nantes, Nantes, France. 5 Cancer Immunology \& Immune Modulation, Boehringer Ingelheim, Ridgefield, Connecticut, USA. ${ }^{6}$ Université de Nantes, CNRS, INSERM, Center for Research in Cancerology and Immunology Nantes-Angers (CRCINA), F-44000 Nantes, France. Immunology Frontier Research Center, Osaka University, Yamada-oka, Suita, Japan. ${ }^{8}$ Université de Rennes, INSERM, CHU Rennes, Institut NUMECAN (Nutrition Metabolisms and Cancer), UMR_S 1241, CRB Santé Rennes, Rennes, France. ${ }^{9}$ Institut des Maladies de l'Appareil Digestif (IMAD), Service d'Hépato-CastroEntérologie et Chirurgie Digestive, ${ }^{10}$ Centre d'investigation Clinique and ${ }^{11}$ Service d'Anatomie et Cytologie Pathologiques, CHU Nantes, Nantes, France.

T cell exclusion causes resistance to cancer immunotherapies via immune checkpoint blockade (ICB). Myeloid cells contribute to resistance by expressing signal regulatory protein- $\alpha$ (SIRP $\alpha$ ), an inhibitory membrane receptor that interacts with ubiquitous receptor CD47 to control macrophage phagocytosis in the tumor microenvironment. Although CD47/ SIRP $\alpha$-targeting drugs have been assessed in preclinical models, the therapeutic benefit of selectively blocking SIRP $\alpha$, and not SIRP $\gamma / C D 47$, in humans remains unknown. We report a potent synergy between selective SIRPa blockade and ICB in increasing memory $T$ cell responses and reverting exclusion in syngeneic and orthotopic tumor models. Selective SIRP $\alpha$ blockade stimulated tumor nest $\mathrm{T}$ cell recruitment by restoring murine and human macrophage chemokine secretion and increased anti-tumor $\mathrm{T}$ cell responses by promoting tumor-antigen crosspresentation by dendritic cells. However, nonselective SIRP $\alpha / S I R P \gamma$ blockade targeting CD47 impaired human T cell activation, proliferation, and endothelial transmigration. Selective SIRP $\alpha$ inhibition opens an attractive avenue to overcoming ICB resistance in patients with elevated myeloid cell infiltration in solid tumors.

\section{Introduction}

Cancer immunotherapy with programmed death-ligand 1 (PDL1)/programmed death-1 (PD-1) antibodies induces robust responses across multiple types of cancers (1-3). However, only a limited number of patients are responsive, in particular those with preexisting $\mathrm{T}$ cell infiltrates and inflammatory signatures in the tumor (4). Tumors in nonresponders typically lack $\mathrm{T}$ cells or have $\mathrm{T}$ cells preferentially located around the tumor region, a process called immune exclusion (5-7). Multiple mechanisms have been associated with resistance to PD-L1/PD-1 therapies, such as

Authorship note: SC, BV, and NP contributed equally to this work as co-senior authors. Conflict of interest: VG, CM, VT, SP, BV, and NP are authors of patents related to SIRP $\alpha / C D 47 / S I R P \gamma$ (W02016/063233, W02017/178653, W02017/068164, W02019/175218). VG, CM, CR, DC, BV, and NP are shareholders of OSE Immunotherapeutics, a company developing SIRP $\alpha$ antagonists (ClinicalTrials.gov NCT03990233). Copyright: (c) 2020, American Society for Clinical Investigation.

Submitted: December 6, 2019; Accepted: August 6, 2020; Published: October 19, 2020 Reference information: / Clin Invest. 2020;130(11):6109-6123.

https://doi.org/10.1172/JCl135528. defective generation of tumor-specific $\mathrm{T}$ cells, impaired formation of memory $\mathrm{T}$ cells, restrained $\mathrm{T}$ cell infiltration, inadequate $\mathrm{T}$ cell function, and local immunosuppression induced by regulatory $\mathrm{T}$ or myeloid cells (8-11). Improved therapy efficacy may therefore be achieved by targeting myeloid cells that positively and negatively orchestrate $\mathrm{T}$ cell responses.

Cells of the myeloid lineage are a major component of the tumor microenvironment (TME) (12). Protumoral M2 macrophages and myeloid-derived suppressive cells (MDSCs) reduce cancer therapy efficacy by inhibiting antitumor $\mathrm{T}$ cell responses or by modulating tumor angiogenesis, survival, spread, and metastasis $(13,14)$. Accumulation of these myeloid cells correlates with poor prognosis and immune checkpoint blockade (ICB) resistance (15-20). Preclinical research and clinical correlational studies suggest that several stromal cell types, such as cancer-associated fibroblast (CAFs), tumor-associated macrophages (TAMs), and MDSCs, prevent T cells from reaching the tumor nest, ultimately limiting the efficacy of ICB (21-24). Conversely, other myeloid cell subsets, such as DCs and antitumoral M1 macrophages, exert antitumor functions, 
including antigen crosspresentation and tumor cell phagocytosis (25-28). However, these processes are limited by CD47 upregulation in tumor cells. CD47 (integrin-associated protein [IAP]) is a ubiquitous membrane protein that binds the innate myeloid immune checkpoint signal regulatory protein- $\alpha$ (SIRP $\alpha$; CD172a or SHPS-1) (29-32). SIRP $\alpha$ is an inhibitory membrane receptor of the immunoglobulin superfamily that is expressed on the surface of myeloid cells in the hematopoietic compartment (33). CD47 is a multifaceted protein that also interacts with SIRP $\gamma$ (a SIRP homolog unique to primates), several integrins, thrombospondin-1 (TSP-1), thrombospondin receptor (CD36), VEGF receptor 2, serpin A1, and Fas (CD95) (34). Binding of CD47 to SIRP $\alpha$ on myeloid cells induces tyrosine phosphorylation of the immunoreceptor tyrosine-based inhibition motif (ITIM) cytoplasmic domain of SIRP $\alpha$, with subsequent recruitment of $\mathrm{SH} 2$-containing tyrosine phosphatase (SHP$1 / 2)$. SHP-1/2 then mediates inhibitory signaling events through protein dephosphorylation, ultimately leading to the inhibition of phagocytosis in macrophages $(33,35)$. High CD47 expression is a mechanism used by malignant cells to evade the immune system that correlates with poor clinical outcomes $(29,36,37)$. Blockade of the CD47-SIRP $\alpha$ interaction with anti-CD47 mAbs promotes tumor elimination by macrophages and decreases cancer cell dissemination in both immunodeficient (T cell lacking) and immunocompetent mice (30, 38-45). Moreover, due to SIRP $\alpha$ 's roles in regulating the cGAS/STING pathway and antigen crosspresentation by DCs $(31,46)$, anti-CD $47 \mathrm{mAbs}$ can also increase crosspriming of $\mathrm{CD}^{+} \mathrm{T}$ cells and induce $\mathrm{T}$ cell-dependent tumor elimination, as shown in preclinical syngeneic rodent models $(30,44)$. However, whether these mechanisms also function when targeting CD47 in humans remains unclear (47). Unlike mice (48), humans and higher primates express SIRP $\gamma$ on T cells. The interaction of SIRP $\gamma$ with CD47 has been associated with cell-cell adhesion, T cell transendothelial migration, and support of $\mathrm{T}$ cell costimulation with DCs $(49,50)$. Preclinical efficacy of CD47/SIRP $\alpha$-targeting drugs has been assessed in vitro in macrophage phagocytosis assays and in vivo using xenograft $\mathrm{T}$ cell immunodeficient mice models (51-56). However, the therapeutic potential of selectively targeting SIRP $\alpha$ to promote $\mathrm{T}$ cell responses in the context of T cell ICB has not been evaluated in higher species.

In this study, we show that selective SIRP $\alpha$ blockade promotes efficient antitumor immune responses and modifies the TME in various syngeneic and orthotopic tumor models. We find a potent synergy between SIRP $\alpha$ blockade and T cell ICB in inducing memory $\mathrm{T}$ cell responses and preventing $\mathrm{T}$ cell exclusion. Moreover, SIRP $\alpha$ blockade allows $\mathrm{T}$ cell recruitment to the tumor nest by restoring macrophage chemokine secretion and increases human antitumor $\mathrm{T}$ cell response by promoting tumor antigen crosspresentation by DCs, as previously described in rodents. In contrast, targeting CD47 or blocking SIRP $\alpha$ and SIRP $\gamma$ simultaneously reduces human $\mathrm{T}$ cell activation, proliferation, and transmigration across the human endothelium, highlighting the importance of selectively targeting SIRP $\alpha$ in humans.

\section{Results}

Anti-mouse SIRP $\alpha$ mAb monotherapy inhibits tumor growth in orthotopic syngeneic models. The preclinical efficacy of SIRP $\alpha$ blockade in immunocompetent mice was previously reported in heterotopic
SIRP $\alpha^{+}$tumor models (57), whereby an antagonist anti-mSIRP $\alpha$ mAb antibody (MY1 clone, ref. 58; rat IgG2a) with Fc-effector functions promoted antibody-dependent cellular cytotoxicity (ADCC) and antibody-dependent cellular phagocytosis (ADCP). To further investigate the importance of target cell opsonization versus SIRP $\alpha$ signaling and interaction with CD47 for therapeutic efficacy in orthotopic tumor mouse models, a mouse Fc IgG1 was used to engineer a MY1 orthosteric inhibitor that blocks SIRP $\alpha$ / CD47 interaction (MY1-G1, Supplemental Figure 1; supplemental material available online with this article; https://doi.org/10.1172/ JCI135528DS1). Comparisons were also performed with a rat IgG1 P84 allosteric inhibitor that blocks SIRP $\alpha$ signaling without preventing its interaction with CD47. In addition, genetically modified SIRP $\alpha$ mutant mice expressing a truncated SIRP $\alpha$ protein that lacks most of the cytoplasmic signaling domain were examined (59).

Two weeks of P84 and MY1-G1 monotherapies initiated 4 days after tumor implantation significantly reduced primary mammary tumor growth and prevented lung metastasis development in the orthotopic 4T1 triple-negative breast cancer model in BALB/c mice (Figure 1, A and B). TIL analysis on day 14 showed an increase

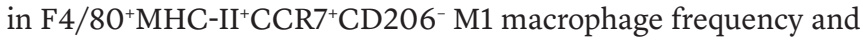
in the M1/M2 (CCR7 $\left.{ }^{-}{ }^{-} 206^{+}\right)$macrophage ratio as well as in NK and memory $\mathrm{T}$ cells (Figure $1 \mathrm{C}$ and Supplemental Figure 2), thereby confirming that TME modification occurred after anti-SIRP $\alpha$ monotherapy. SIRP $\alpha$ blockade also modified peripheral immune responses, as revealed by the increased frequency of central memory $\mathrm{CD} 44^{+} \mathrm{CD} 62 \mathrm{~L}^{+} \mathrm{CD} 4^{+} \mathrm{T}$ lymphocytes in draining lymph nodes, a higher M1/M2 ratio, and a reduction in the frequency of Tregs $\left(\mathrm{CD} 4^{+} \mathrm{CD} 25^{+} \mathrm{Foxp}^{+} \mathrm{CD} 127^{\mathrm{lo}}\right)$ in the spleen. Surgical resection of primary $4 \mathrm{~T} 1$ mammary tumors after 10 days of treatment confirmed the significant reduction in tumor spread and metastasis. Indeed, transient SIRP $\alpha$ blockade with P84 or MYI-G1 resulted in $71 \%$ survival when compared with $14 \%$ survival in the isotype control group (Figure 1D). Moreover, MY1-G1 administration in the AK7 orthotopic mesothelioma C57BL/6 mouse model significantly prolonged survival (Figure 1E). Finally, mutant mice lacking SIRP $\alpha$ signaling and injected with AK7 mesothelioma tumor cells exhibited prolonged survival by $55 \%$, when compared with WT animals. Interestingly, surviving SIRP $\alpha$ mutant mice were also protected against a second AK7 heterotopic tumor challenge in the peritoneum, suggesting that inhibition of SIRP $\alpha$ signaling produces a robust and durable antitumoral response (Figure $1 \mathrm{~F}$ ).

Anti-mouse SIRPa mAbs induce durable adaptive immune responses in combination with $T$ cell immune checkpoint agents. Preclinical syngeneic models with suboptimal response to $\mathrm{T}$ cell ICB were used to assess the efficacy of combined immunotherapies targeting both the innate and adaptive immune checkpoints. Monotherapy with anti-PD-L1 or MY1-G1 anti-mSIRP $\alpha$ mAb significantly inhibited tumor growth in immunocompetent mice implanted subcutaneously with the colon adenocarcinoma MC38 cell line, although a complete response (CR) was only observed in $15 \%-30 \%$ of mice (Figure 2A). However, the combination of MY1-G1 and anti-PD-L1 mAbs strongly inhibited tumor growth in all mice and induced durable CR in $79 \%$ of mice. After complete elimination of the drugs, $90 \%$ of the cured mice were resistant to a secondary tumor challenge with the same cell line, suggesting that combined anti-mSIRP $\alpha$ and anti-PD-L1 mAb therapy induces 


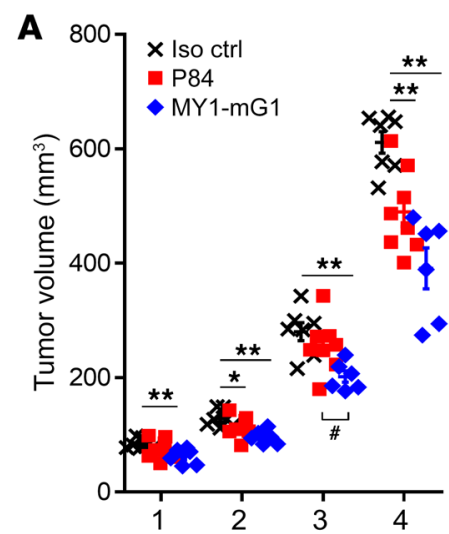

Weeks after tumor injection
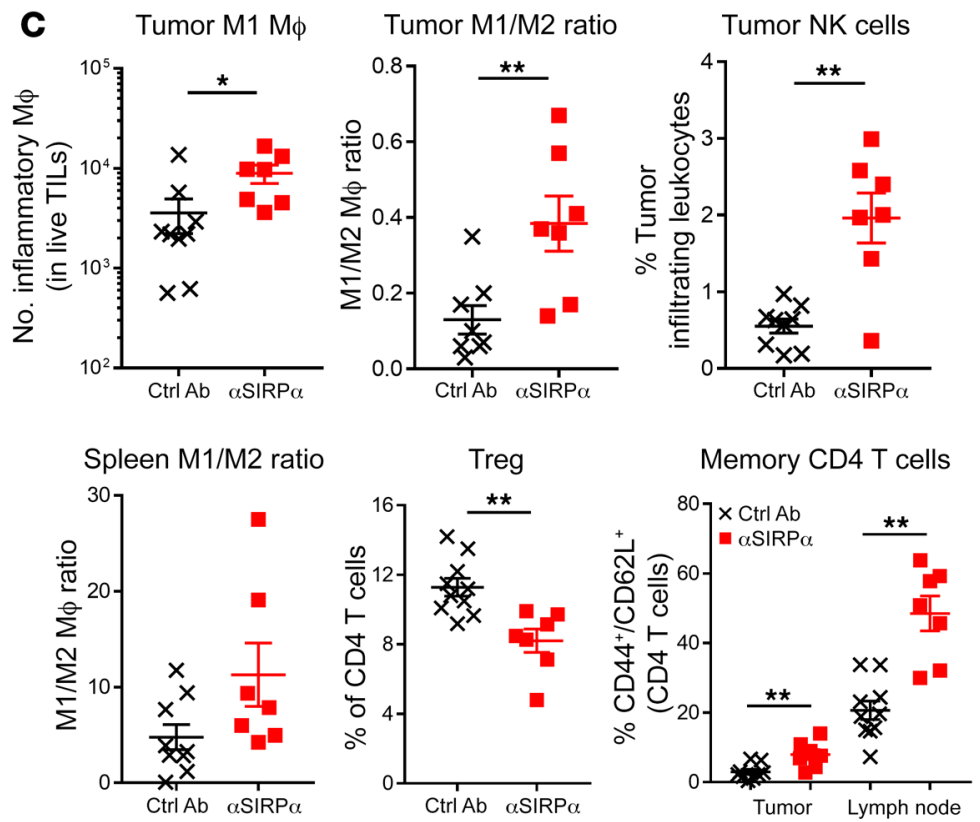

D

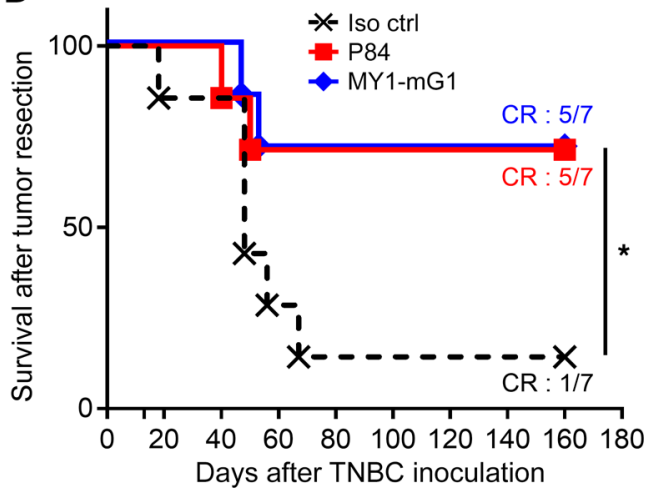

E

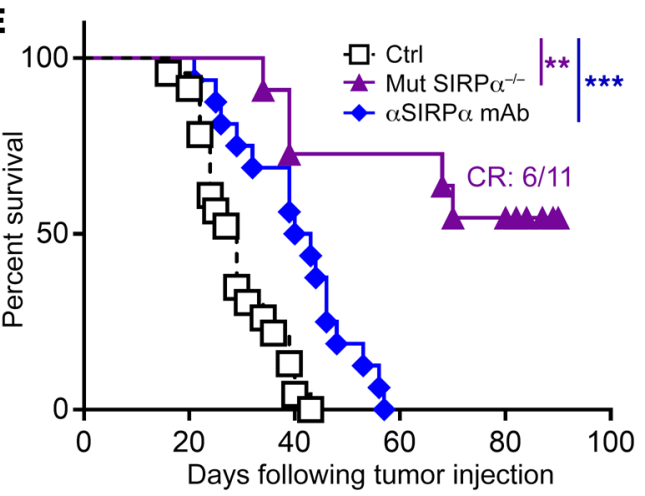

$\mathbf{F}$

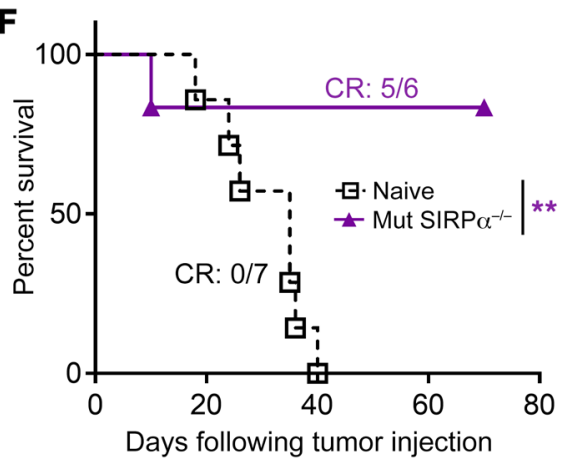

Figure 1. Anti-SIRPa monotherapy efficacy in orthotopic syngeneic tumor models. (A) Primary tumor volume and (B) lung metastasis count in the 4T1 triple-negative breast orthotopic tumor model $\left(0.25 \times 10^{6}\right.$ cells injected in the mammary fat gland) of mice treated i.p. with a control mAb (black, $n=7$ ), P84 (red, $n=8$ ), or MY1-G1 (blue, $n=6$ ) anti-SIRP $\alpha$ antagonist mAbs at $10 \mathrm{mg} / \mathrm{kg} 3$ times from day 4 to day 18. (C) Tumor-infiltrating frequencies among live cells and peripheral leukocyte phenotype modification after treatment with P84 (red, $n=7$ ) or control (black, $n=9$ ) mAbs 15 days after tumor implantation. (D) Same protocols and symbols as in A, but a surgical resection of the primary tumor was performed 13 days after tumor implantation and the survival of mice ( $n=7$ per group) was analyzed. (E) Survival of WT (black, $n=23$ ) and SIRP $\alpha$ mutant (purple, $n=11$ ) untreated mice injected on day 0 in the pleural cavity with AK7 mesothelioma tumor cells $\left(3 \times 10^{6}\right)$. Some WT mice were also treated i.p. with the MY-1-mG1 anti-SIRP $\alpha$ mAb $(b l u e, n=16)$ from day 4 to day 32 at $10 \mathrm{mg} / \mathrm{kg} 3$ times a week. Three independent experiments were performed. (F) Cured-SIRP $\alpha$ mutant mice from the AK7 model were rechallenged i.p. with a new load of $3 \times 10^{6}$ AK7 cells $(n=6)$. The same injection was performed in untreated WT mice as control $(n=7)$. ${ }^{*} P<0.05 ;{ }^{* *} P<$ 0.01 ; ${ }^{* *} P<0.005$, compared with control group; ${ }^{*} P<0.05$ for P84 and MY-1 comparison, unpaired Mann-Whitney $U$ test or log-rank for survival.

memory immune responses (Figure 2B). Similarly, monotherapy with P84 anti-mSIRP $\alpha$ mAb, anti-PD-L1 blocking $\mathrm{mAb}$, or anti-4$1 \mathrm{BB}$ agonist $\mathrm{mAb}$ significantly prolonged survival in the CD47/ PD-L1 expressing orthotopic Hepa1.6 hepatocellular carcinoma (HCC) syngeneic model, albeit with low CR rates (0\%, 7\%, and $26 \%$, respectively; Figure 2C and Supplemental Figure 3). On the other hand, treatment with P84 anti-mSIRP $\alpha$ mAb combined with either anti-PD-L1 or anti-41BB induced durable CRs in $61 \%$ and $80 \%$ of mice, respectively. MY1-G1 anti-mSIRP $\alpha$ combined with a PD-L1 antagonist or 4-1BB agonist induced durable CR in
$100 \%$ of mice (Supplemental Figure 4, A and B). Moreover, after the treatment was completed, all of the cured mice successfully rejected a second Hepa1.6 tumor challenge in the absence of any treatment (Figure 2D and Supplemental Figure 4C). Intravenous adoptive transfer of spleen T lymphocytes $\left(2.5 \times 10^{6}\right)$ or whole isolated tumor leukocytes $\left(2 \times 10^{6}\right)$ from anti-mSIRP $\alpha+$ anti$4-1 \mathrm{BB}$ cured mice significantly protected naive and untreated mice after Hepa1.6 orthotopic implantation, further suggesting that this combined treatment induces robust $\mathrm{T}$ cell-mediated memory immune responses (Figure $2 \mathrm{E}$ ). These results were 


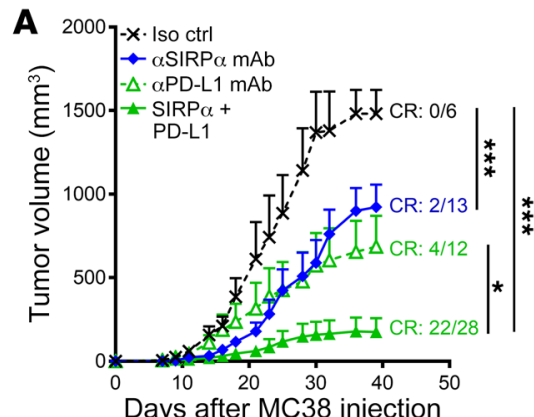

Days after MC38 injection

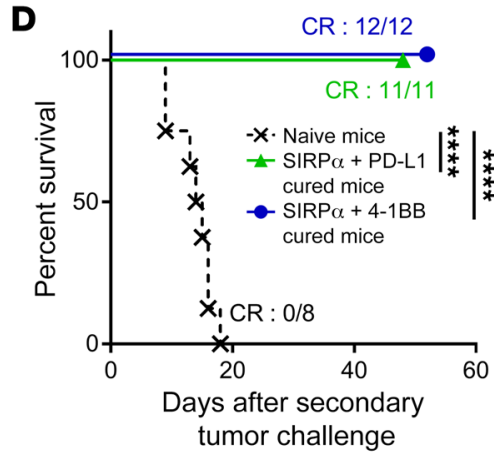

B

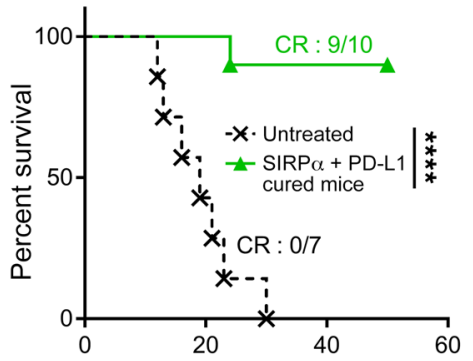

Days after secondary tumor challenge

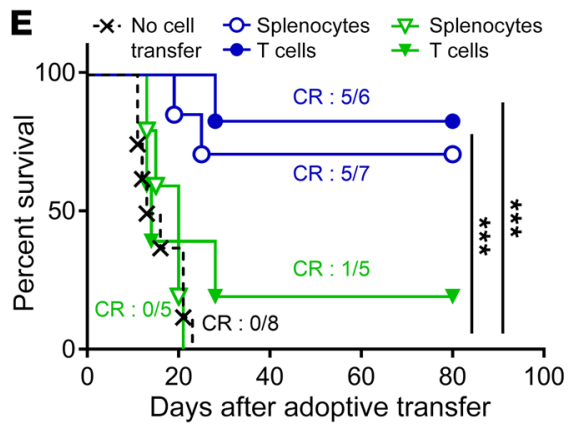

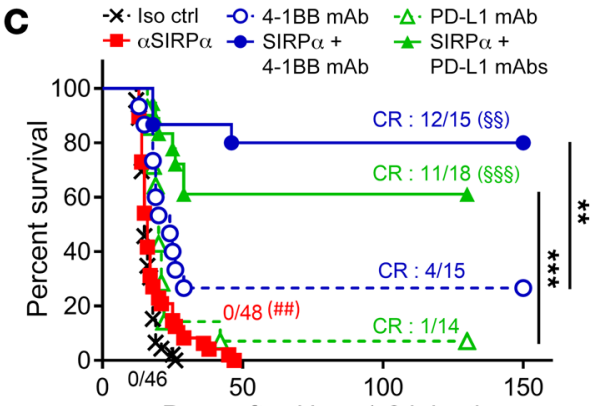

Days after Hepa1.6 injection

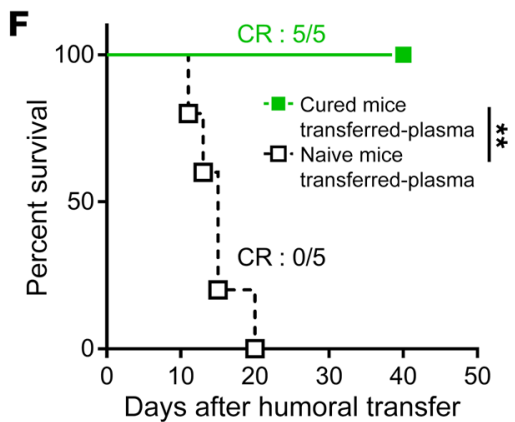

G

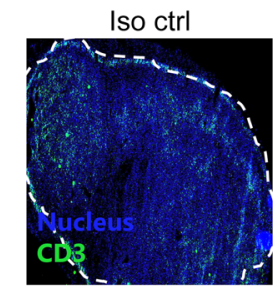
$\alpha \operatorname{SIRP} \alpha$
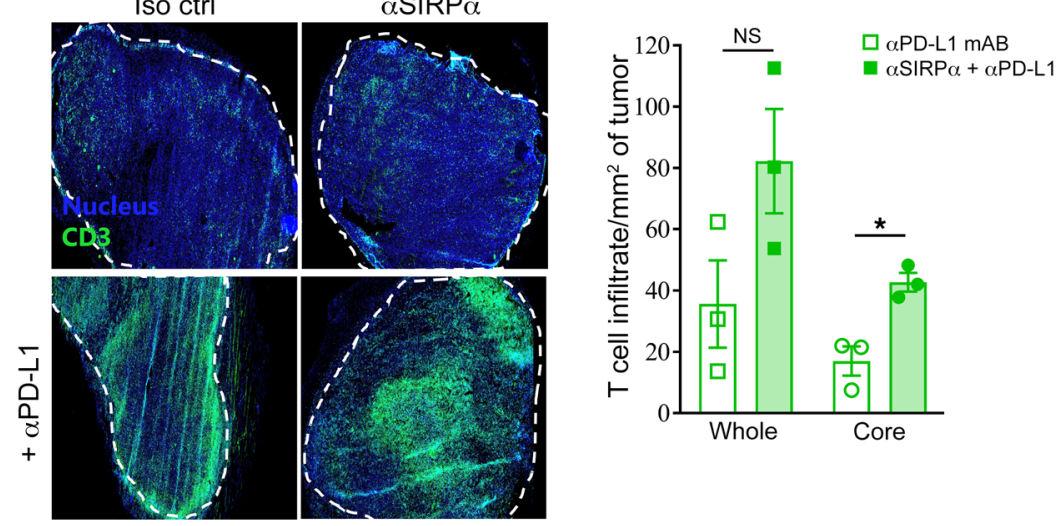

H
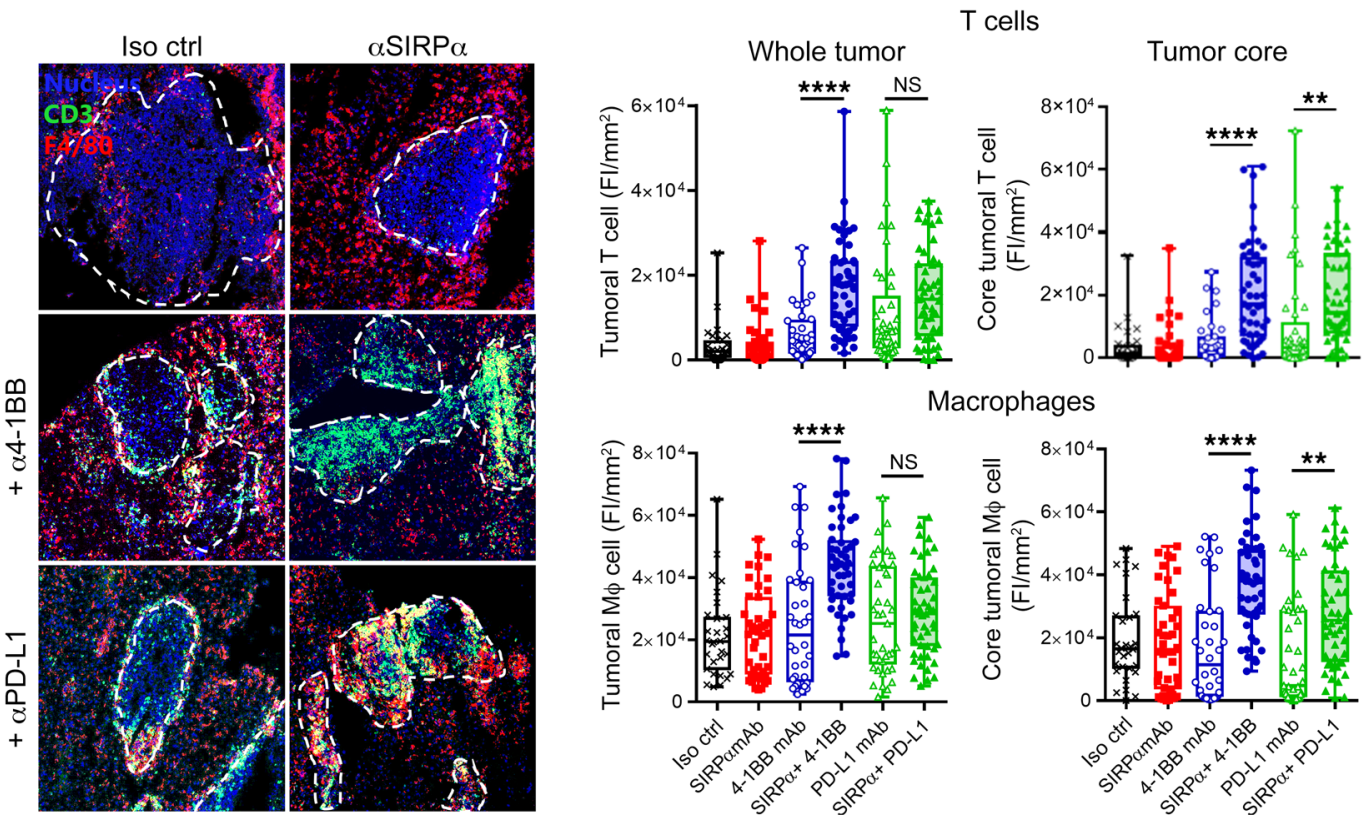
Figure 2. Anti-SIRP $\alpha$ synergizes with anti-PD-L1 and anti-4-1BB mAbs and prevents $T$ cell exclusion. (A) Tumor volume in MC38 model of mice treated i.p. triweekly (days 4-28) with control (black, $n=6$ ), MY1-G1 anti$\mathrm{SIRP} \alpha$ (blue, $10 \mathrm{mg} / \mathrm{kg}, n=13$ ), anti-PD-L1 (10F-9G2, open green triangle, $6 \mathrm{mg} / \mathrm{kg}, n=12$ ), or MY1-G1 plus anti-PD-L1 (filled green triangle, $n=28$ ) mAbs. (B) Survival of untreated naive (black, $n=7$ ) or MC38 tumor-free mice treated with the anti-SIRP $\alpha+\mathrm{PD}-\mathrm{L} 1$ combination (green, $n=10$ ) and rechallenged with MC38 cells 2 months after first tumor inoculation. (c) Survival in the Hepa1.6 HCC orthotopic model of mice treated i.p. (days 4-28) triweekly with control (black, $n=48$ ), P84 anti-SIRP $\alpha$ (red, $10 \mathrm{mg} / \mathrm{kg}, n=48$ ), anti-PD-L1 (open green triangle, $6 \mathrm{mg} / \mathrm{kg}, n=14$ ), or P84+anti-PDL-1 (filled green triangle, $n=18$ ) mAbs. Some mice received 2 injections (days 4 and 8 ) of $3 \mathrm{mg} / \mathrm{kg} 3 \mathrm{H} 3$ (4-1BB agonist; open blue circle, $n=15$ ) alone or in combination with P84 (filled blue circle, $n=15$ ). Three independent experiments are represented. (D) Survival of untreated naive or Hepa1.6-cured mice rechallenged with intrasplenic Hepa 1.6 cells 3 to 4 months after first tumor inoculation. Symbols are the same as in C. (E) Survival of untreated mice implanted with Hepa 1.6 cells in the portal vein after adoptive transfer of $10 \times 10^{6}$ splenocytes or $2.5 \times 10^{6}$ spleen T cells isolated from cured mice in $\mathbf{D}$. (F) Same as in $\mathbf{E}$ after adoptive transfer of $200 \mu \mathrm{L}$ of plasma from PD-L1/SIRP $\alpha$-cured mice. (C) T cell infiltrates histological quantification in the entire tumor or in the core $50 \%$ of the tumor from the center) in the MC38 model 3 weeks after tumor inoculation. Treatment was same as in A. Blue, nucleus; green, CD3. (H) T cell and macrophage infiltrate histological quantification in the Hepa1.6 model 2 weeks after tumor inoculation. $\mathrm{Fl}$, fluorescent intensity. Treatment was same as in C. Blue, nucleus; green, CD3; red, F4/80. Original magnification, $\times 20 .{ }^{*} P<0.05,{ }^{* *} P<0.01$ or ${ }^{\# \#} P<0.01$, ${ }^{* *} P<0.005$, ${ }^{* * *} P<0.001$, compared with control group; $\$ \$ P<0.01,{ }^{\S \S} P<0.005$ for T cell monotherapies compared with combination, unpaired Kruskall-Wallis test, Mann-Whitney $U$ test, or log-rank test or survival.

corroborated by similar adoptive transfer experiments in SIRP $\alpha$ mutant mice treated with anti-4-1BB mAbs (Supplemental Figure 5). Surprisingly, while anti-mSIRP $\alpha+$ anti-PD-L1 cured mice displayed tumor-specific spleen memory $\mathrm{T}$ cells responses ex vivo (Supplemental Figure 6A), adoptive transfer of spleen T cells from these mice had no significant impact on survival (Figure 2E). Nonetheless, adoptive intravenous transfer of plasma from anti$\mathrm{mSIRP} \alpha+$ anti-PD-L1 cured mice protected naive untreated mice (Figure 2F). Indeed, mice initially treated with anti-PD-L1 alone or in combination with anti-mSIRP $\alpha$ exhibited plasmatic antiHepa1.6 and anti-hepatocyte IgG responses (Supplemental Figure 5, B and C). Tumor-infiltrating leukocyte (TIL) analysis after 10 days of treatment confirmed that the combination of SIRP $\alpha$ blockade with anti-PD-L1 or anti-4-1BB modified the TME (Supplemental Figures 7-9). Specifically, both these combination therapies significantly increased T cell frequency $(P<0.05)$ in the TME and reduced the frequency of immunosuppressive Tregs and MDSCs. However, while combining anti-mSIRP $\alpha \mathrm{mAb}$ with anti-4-1BB $\mathrm{mAb}$ favored $\mathrm{CD} 8^{+} \mathrm{T}$ cells, the combination of anti-mSIRP $\alpha$ with anti-PD-L1 favored CD4 ${ }^{+} \mathrm{T}$ cells responses. Moreover, SIRP $\alpha$ / PD-L1 combined therapy significantly reduced the frequency of immature transitional B cells $(P<0.01)$, but increased plasmablast infiltration $(P<0.05)$.

Anti-mouse and anti-human SIRPa mAbs promote chemokine secretion and $T$ cell migration in the tumor nest. Tumor-associated stromal cells (e.g., macrophages) have been recently implicated in the immune exclusion phenotype observed in a subcutaneous MC38 tumor model (21). In agreement with these data, we found that after anti-PD-L1 therapy, $\mathrm{CD}^{+} \mathrm{T}$ cells weakly infiltrated the tumor nest in the MC38 model (Figure 2G). In contrast, SIRP $\alpha$ blockade in combination with $\mathrm{T}$ cell $\mathrm{ICB}$ induced $\mathrm{T}$ cell infiltration in the tumor nest, which is consistent with the observed high rates of CR (Figure 2A). In the orthotopic Hepa1.6 model, T cell infiltration increased in the liver after 10 days of treatment with anti-PD-L1 or anti-4-1BB monotherapy. However, these T cells were mainly on the margin of tumor nodules in regions where macrophages could also be found (Figure $2 \mathrm{H}$ ). Similarly to what was seen with the MC38 model, anti-mSIRP $\alpha+$ anti-PD-L1 or anti-4-1BB combinations significantly increased $\mathrm{CD}^{+} \mathrm{T}$ cell and $\mathrm{F} 4 / 80^{+}$macrophage infiltration within tumor nodules (Figure $2 \mathrm{H}$ ). Nanostring transcriptomic analysis in the liver (murine PanCancer Immune Profiling [PCIP] panel) revealed that both SIRP $\alpha / P D-L 1$ and SIRP $\alpha / 4-1 B B$ combination treatments modified the TME extensively (Figure 3A and Supplemental Figure 10) and induced similar changes in gene expression. STRING analysis of the upregulated gene cluster identified increased expression of genes associated with chemokine and cytokine pathways (Figure $3, \mathrm{~B}$ and $\mathrm{C}$ ) and downregulation of genes involved in metastasis and the TGF- $\beta$ pathway (Supplemental Figure 11).

Histological and transcriptomic analyses of the TME in our mouse models seems to indicate that, in addition to inhibiting phagocytosis, SIRP $\alpha$ might also regulate other immune functions, such as those related to chemotaxis and immune cell activation in a CD47-expressing TME. Indeed, anti-mSIRP $\alpha$ mAbs appear to reinvigorate tumor-associated myeloid cells and promote $\mathrm{T}$ cell attraction to the tumor nest. To assess whether this SIRP $\alpha$ function is conserved in humans, surgically dissociated tumor explants from HCC $(n=7)$, colorectal carcinoma (CRC) $(n=1)$, pancreatic ductal carcinoma (PDAC) $(n=1)$, and renal cell carcinoma (RCC, $n=2)$ patients as well as cells of mesothelioma pleural effusion (Meso) $(n=7)$ were cultured ex vivo with a selective antagonist human anti-SIRP $\alpha \mathrm{mAb}$ (OSE-172, binds preferentially the main SIRP $\alpha$ V1 variant, but not SIRP $\gamma$ [Supplemental Figures 12, 13A, and 14] and promotes tumor cell phagocytosis [Supplemental Figure 15]) or an isotype control mAb (Figure 4A). Transcriptomic NanoString analysis (human PCIP panel) conducted after 48 hours revealed that selective anti-hSIRP $\alpha$ mAb alone significantly modified gene expression in a variety of human TMEs (Figure $4 \mathrm{~B}$ and Supplemental Figure 16). Notably, selective anti-hSIRP $\alpha$ mAbs induced overexpression of genes involved in chemotaxis and adaptive immune functions (Figure 4C). To assess whether chemokine gene expression upregulation induced by SIRP $\alpha$ inhibition is related to tumor-associated myeloid cell modification, isolated CD14 ${ }^{+}$myeloid cells from human ovarian cancer ascites $(n$ $=8$ ) were cultured ex vivo for 48 hours with selective anti-hSIRP $\alpha$ or isotype mAbs (Figure 4D). Transcriptomic (NanoString Human MII_v2 panel) and Gene Set Enrichment Analysis (GSEA) showed that selective SIRP $\alpha$ blockade significantly enriches chemokine and cytokine gene expression in myeloid cells when compared with the isotype control condition (Figure 4E). Consistent with this, clustering of the 100 most overexpressed genes in antihSIRP $\alpha$ versus isotype conditions identified 2 clusters of genes associated with chemokine and cytokine functions (Figure 4F).

To confirm that SIRP $\alpha$ negatively controls chemokine secretion and hence $\mathrm{T}$ cell recruitment, we performed a series of in vitro and in vivo experiments using human macrophages cultured with 
A
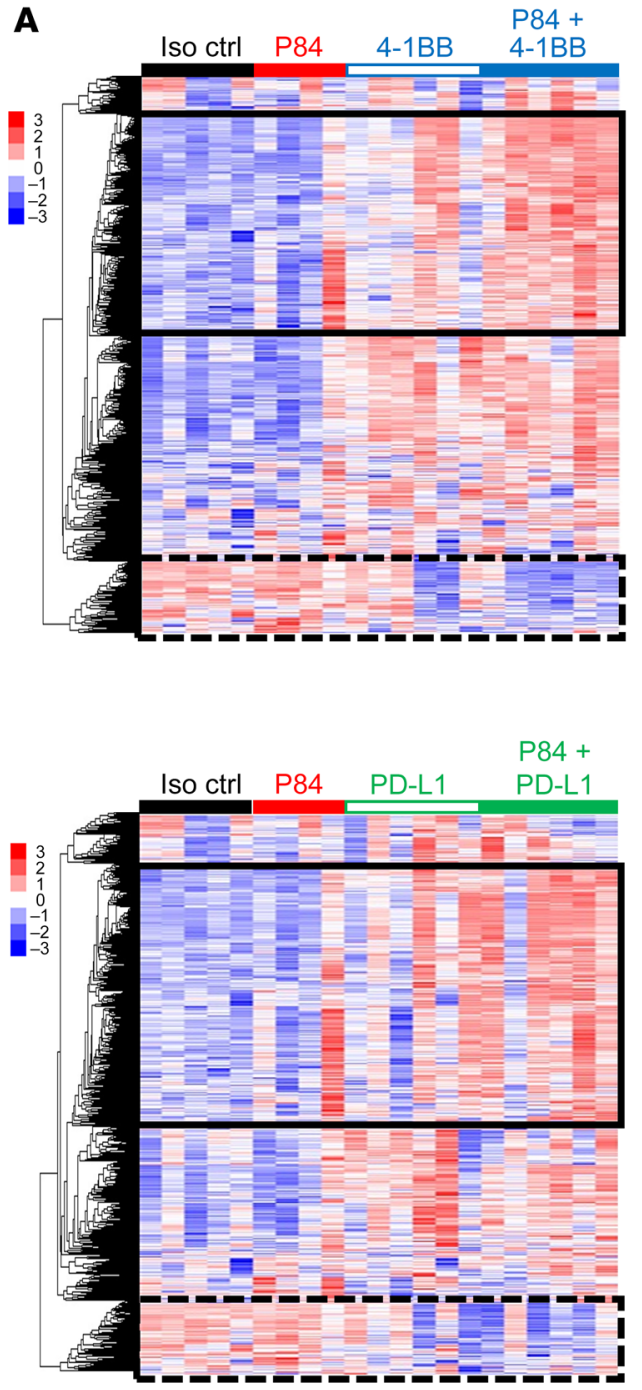

B
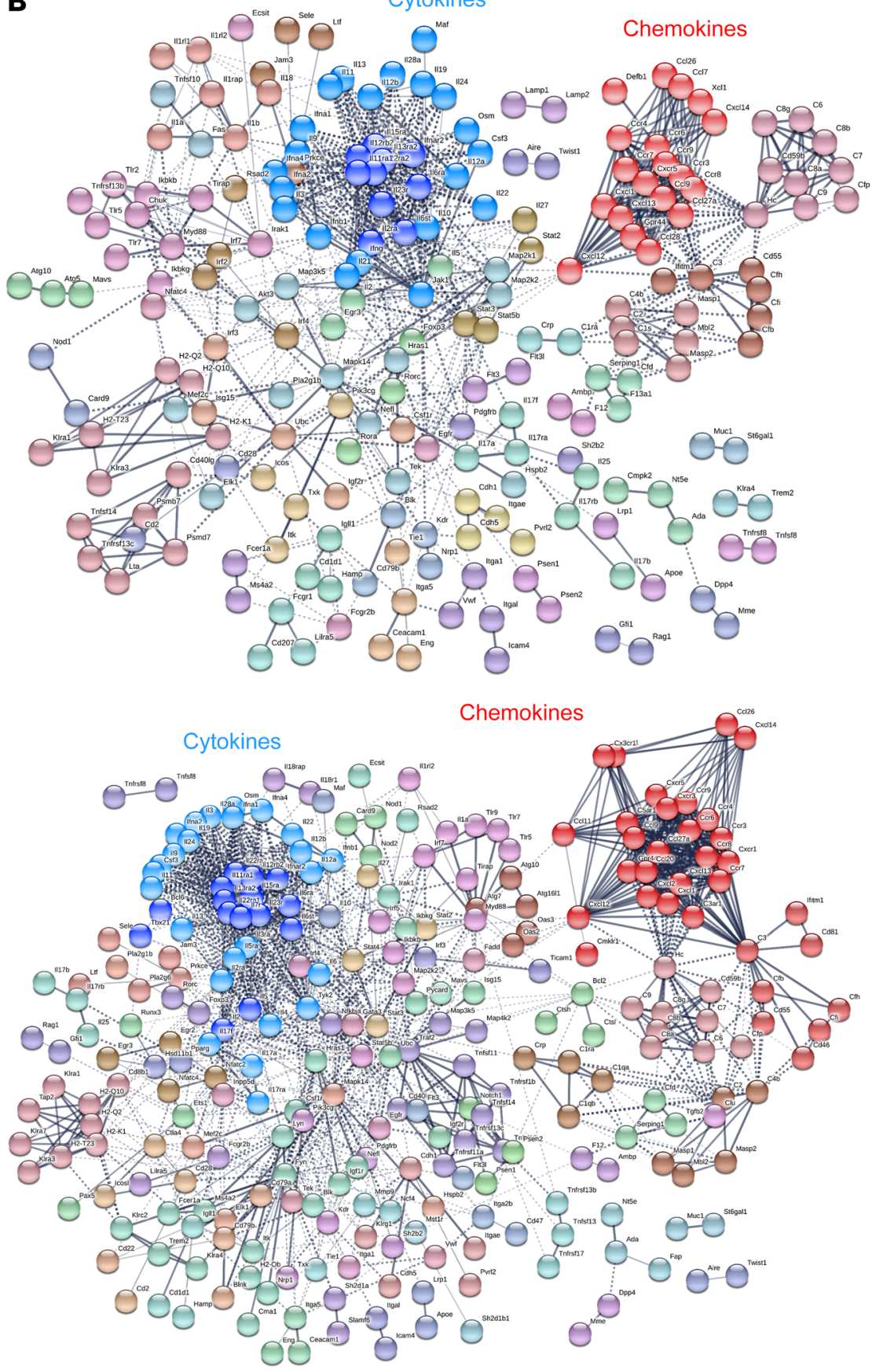

C

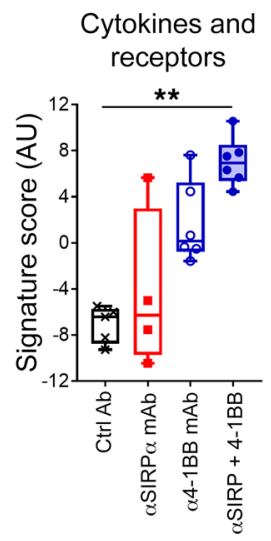

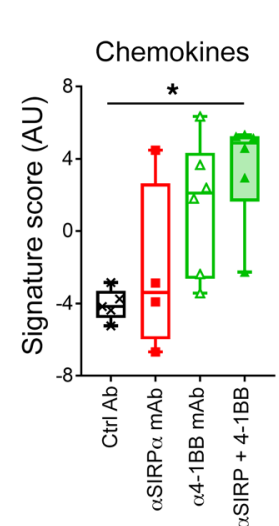


Figure 3. TME transcriptomic modifications after anti-SIRP $\alpha$ combination therapy in mouse. Transcriptomic NanoString (PCIP panel) analysis of the liver of mice in the orthotopic Hepa1.6 HCC model 10 days after treatment initiation. Colors and symbols are the same as in Figure 2C. (A) nSolver relative gene expression analysis and heatmap representation (normalized to the median of control group) of mice treated with control Ab $(n=5)$, P84 anti-SIRP $\alpha$ mAb $(n=4), 4-1 \mathrm{BB}$ agonist \pm P84 (upper panel, $n=6$ /group), or anti-PD-L1 mAb \pm P84 (lower panel, $n=6$ /group). The solid-line rectangle surrounds the cluster of gene expression increased with P84 combination, while the dotted-line rectangle surrounds the cluster of gene expression decreased with P84 combination (see Supplemental Figure 8 for detailed list). (B) STRING protein-protein network analysis of upregulated gene cluster surrounded by solid-line rectangle in A. (C) Transcriptomic gene expression signature as annotated by NanoString according to the related function obtained using nSolver advanced analysis. ${ }^{*} P<0.05$; ${ }^{* *} P<0.01$, unpaired Kruskall-Wallis test.

human tumors and human T lymphocytes. We first confirmed in vitro that CD47 transactivation of SIRP $\alpha$ inhibits chemokine secretion by human macrophages independently of tumor phagocytosis and that anti-hSIRP $\alpha$ mAbs restored this CD47-induced reduction in chemokine secretion (e.g., CCL3, CCL4) (Figure 5A). Similarly, selective SIRP $\alpha$ blockade significantly increased CCL4 secretion by human monocytes cultured with different CD47-expressing human tumor cell lines (Figure 5B and Supplemental Figure 17). To assess whether human macrophages immunosuppressed by SIRP $\alpha$ signaling affect T cell migration in vivo, we injected monocyte-derived human macrophages intradermally in the ears of immunodeficient mice previously infused i.p. with autologous human peripheral blood leukocytes (Figure 5C). Ear immunohistological analysis performed 6 hours after macrophage injection showed that human $\mathrm{T}$ cell infiltration was significantly increased by selective anti-hSIRP $\alpha$ mAbs (Figure 5, D and E). Interestingly, although a nonselective antagonist anti-hSIRP $\alpha / \gamma \mathrm{mAb}$ (Kwar23, binds to both hSIRP $\alpha$ and hSIRP $\gamma$ and blocks their interaction with CD47; Supplemental Figures 13 and 14) restored chemokine secretion in human macrophages in vitro (Supplemental Figure 18A), $\mathrm{T}$ cell migration in vivo was not affected by this SIRP $\alpha / \gamma$ antagonist (Figure 5, D and E). To evaluate whether SIRP $\alpha / \gamma$ also differentially regulates human $\mathrm{T}$ cell migration within human tumors expressing CD47, we quantified $\mathrm{T}$ cell migration within an established human tumor spheroid model as previously described in the literature $(60,61)$ using a A549 NSCLC cell line cultured for 3 days with human monocytes and MRC-5 fibroblasts (Figure 5F). As observed in vivo in humanized mice, selective SIRP $\alpha$ blockade significantly increased human $\mathrm{T}$ cell migration within human tumor spheroids, while concomitant blockade of SIRP $\alpha$ and SIRP $\gamma$ decreased it as compared with isotype control condition (Figure 5, G and H). These data are consistent with previous research suggesting that SIRP $\gamma$ plays an important role in cell-cell adhesion and/or $\mathrm{T}$ cell transendothelial migration in human T lymphocytes $(49,50)$.

Selective targeting of human SIRP $\alpha$ without affecting SIRP $\gamma /$ CD47 interaction increases human $T$ cell responses. Recently, it has been shown that SIRP $\alpha / C D 47$ blockade using $C d 47^{-/-}$mutant mice or anti-CD47 mAbs (which could opsonize tumor cells) promotes antigen crosspresentation by DCs and, consequently, antigen-specific $\mathrm{T}$ cell activation $(31,46)$. Notably, we found that while type 2 conventional DCs $\left(\mathrm{cDC} 2, \mathrm{CD} 11 \mathrm{c}^{+} \mathrm{CD} 11 \mathrm{~b}^{+} \mathrm{CD} 8^{-}\right)$express high levels of SIRP $\alpha, \mathrm{cDC} 1 \mathrm{~s}\left(\mathrm{CD} 11 \mathrm{c}^{+} \mathrm{CD} 11 \mathrm{~b}^{\mathrm{lo}} \mathrm{CD} 8^{+}\right)$express very low levels of the receptor (Supplemental Figure 19A). Consistent with these SIRP $\alpha$ expression patterns, cDC1s were more efficient than cDC2s in promoting antigen crosspriming of OT-I CD8 ${ }^{+} \mathrm{T}$ cells in vitro (Supplemental Figure 19B). Moreover, anti-mSIRP $\alpha$ (P84, MY1-G1) and anti-CD47 mAbs significantly increased OT-I T cells crosspriming by $\mathrm{cDC} 2$, but not with $\mathrm{cDC1}$ (Supplemental Figure 19C). To determine a potential function of SIRP $\alpha$ in promoting antigen crosspriming of OT-I CD $8^{+} \mathrm{T}$ cells in vivo, anti-mSIRP $\alpha-$ treated (MY1-G1) mice were infused intravenously with OT-I CD8 ${ }^{+}$ T cells. At 48 hours after i.p. challenge with OVA antigen, these mice overexpressed class I MHC, CD86 costimulatory molecule, and CD103 integrin in both $\mathrm{CDC} 1$ and $\mathrm{CDC} 2$ subsets (Supplemental Figure 19D). Moreover, proliferation of OT-I T cells was significantly increased in vivo after anti-mSIRP $\alpha$ mAb (MY1-G1) treatment $(P<0.01)$ (Supplemental Figure 19, E and F), confirming that inhibiting SIRP $\alpha$ signaling increases $C D 8^{+} \mathrm{T}$ cell crosspriming by myeloid cells, as previously described in mice (46).

The specific expression (Supplemental Figures 20-23) and the potential role of the SIRP $\gamma$ homologue (Supplemental Figure 24) in human $\mathrm{T}$ cell biology raise the question of whether the benefit of anti-SIRP $\alpha$ antibodies and CD47-targeting drugs in increasing $\mathrm{T}$ cell activity in mice translates similarly to humans. To test this, human tumor melan-A-specific HLA-A2 ${ }^{+} \mathrm{CD} 8^{+}$cytotoxic $\mathrm{T}$ cell clones (melan-A-specific cytotoxic T lymphocyte [CTL]) isolated from melanoma patients (62) were restimulated with HLA-A2 ${ }^{+}$monocyte-derived human DCs loaded with a melan-A 25-mer long peptide. We found that incubation with a selective anti-hSIRP $\alpha$ (OSE-172) mAb significantly increased IFN- $\gamma$ secretion by human melan-A-specific CTL, whereas a nonselective anti-hSIRP $\alpha / \gamma \mathrm{mAb}$ (Kwar23) and different anti-CD47 mAbs did not (Figure 6, A and B). Interestingly, the nonselective anti$\mathrm{hSIRP} \alpha / \gamma \mathrm{mAb}$ prevented the beneficial effect of OSE-172 in promoting IFN- $\gamma$ secretion, suggesting that blocking SIRP $\gamma$ reduces $\mathrm{T}$ cell crosspriming responses. To understand the mechanisms underlying this process, we tested these antibodies using a mouse thymoma T cell line lacking SIRP $\gamma$ expression and transfected with the TCR of melan-A-specific human CTL and human CD8. Notably, both the selective anti-hSIRP $\alpha \mathrm{mAb}$ alone and the combination of anti-hCD47 + anti-mCD47 mAbs increased T cell response when the thymoma $\mathrm{T}$ cells were cocultured with $\mathrm{HLA}-\mathrm{A} 2^{+}$monocyte-derived human DCs loaded with the melan-A 25-mer long peptide (Figure $6 \mathrm{C}$ ). Taken together, these results demonstrate that, while blocking SIRP $\alpha$ increases antigen-specific crosspresentation by human DCs, blocking the SIRP $\gamma / C D 47$ interface decreases the crosspriming response of human T lymphocytes. In addition, targeting CD47 on human T cells with anti-CD47 mAbs also inhibited conventional anti-CD3 + anti-CD28 polyclonal stimulation, allogeneic stimulation, mixed lymphocyte reaction, and transendothelial T cell migration (Figure 6, D-G).

\section{Discussion}

ICB therapies targeting $\mathrm{T}$ cells show broad clinical efficacy in oncology; however, medical needs remain high, as up to $70 \%$ of patients do not show a long-term response, with the exception of those with some rare indications. Combination therapies using 2 types of ICB targeting T cells have already demonstrated 
A

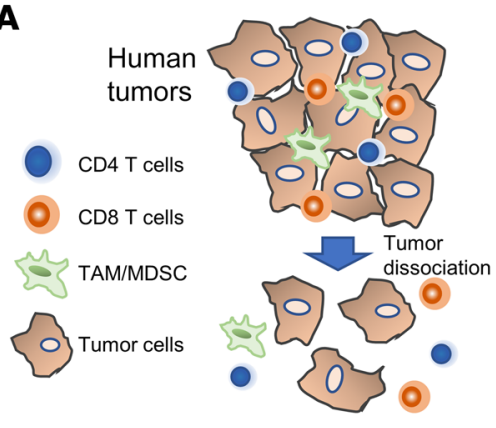

48h incubation $\alpha$ SIRP $\alpha$

Transcriptomic analysis (Nanostring PCIP panel)
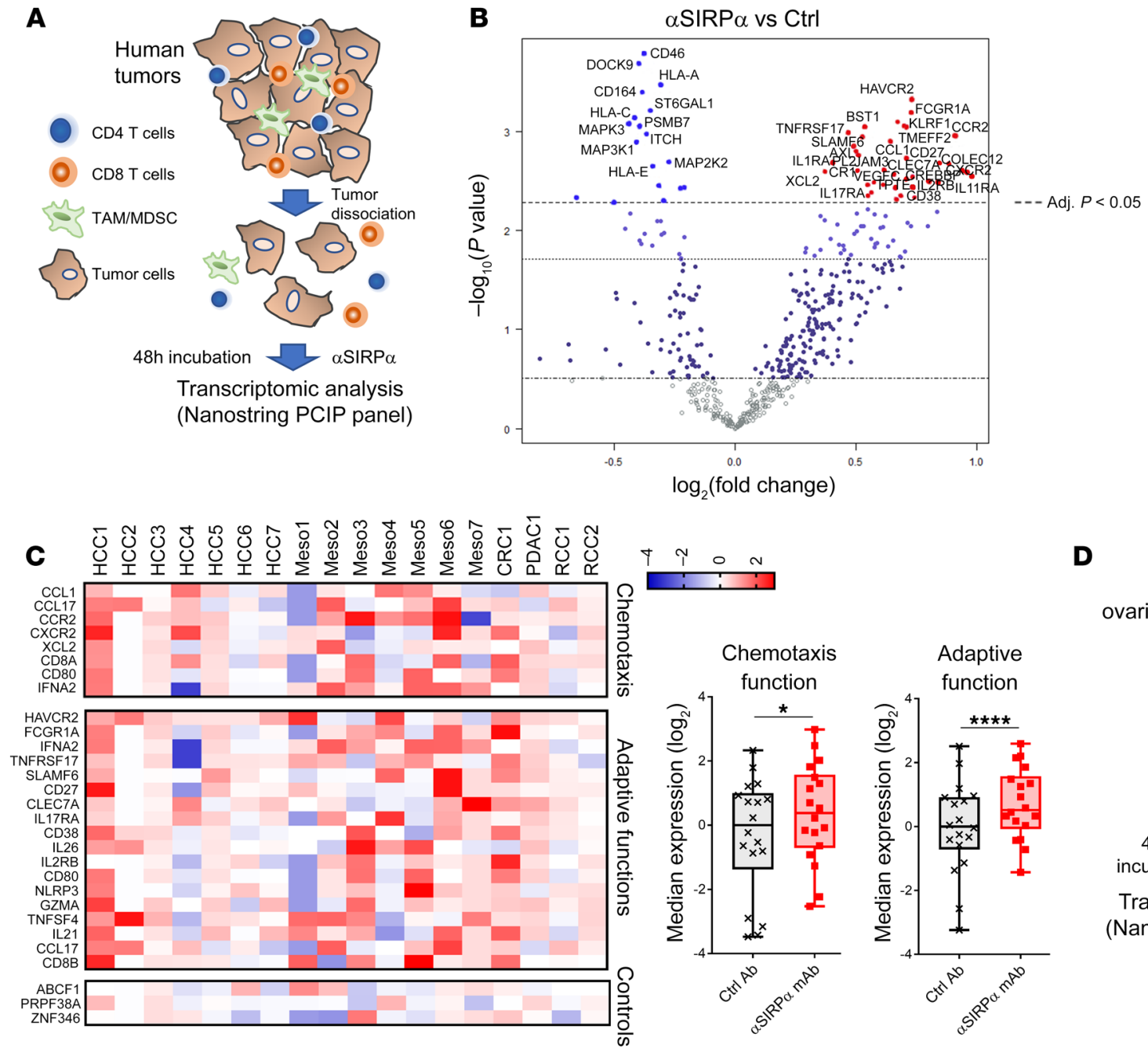

D

Ascites from
ovarian carcinoma patients
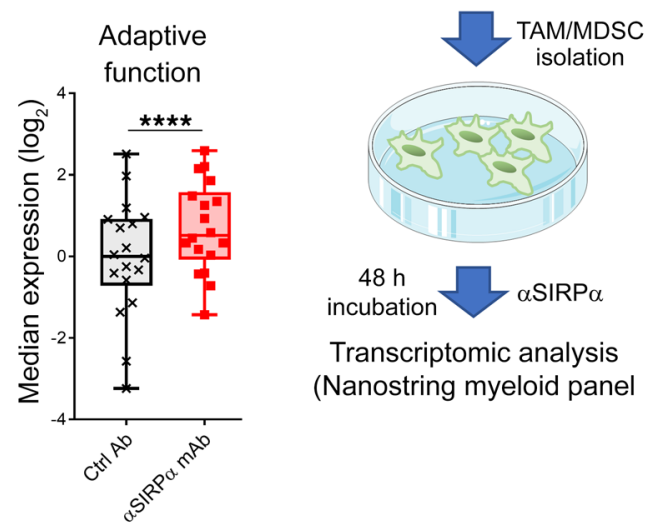

Transcriptomic analysis (Nanostring myeloid panel
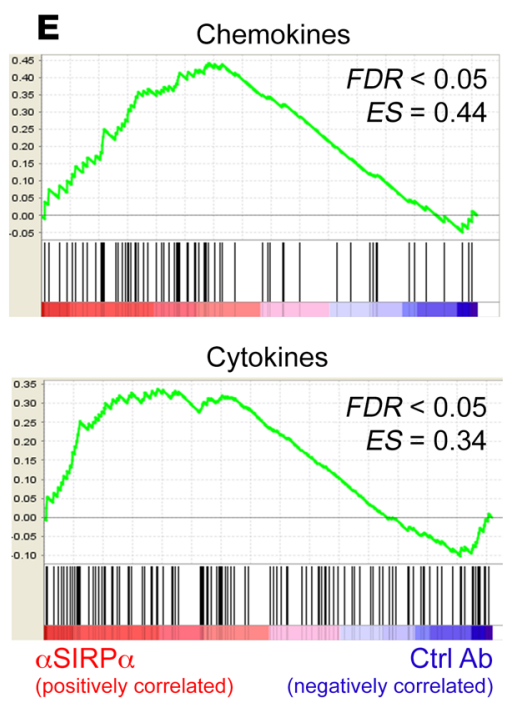
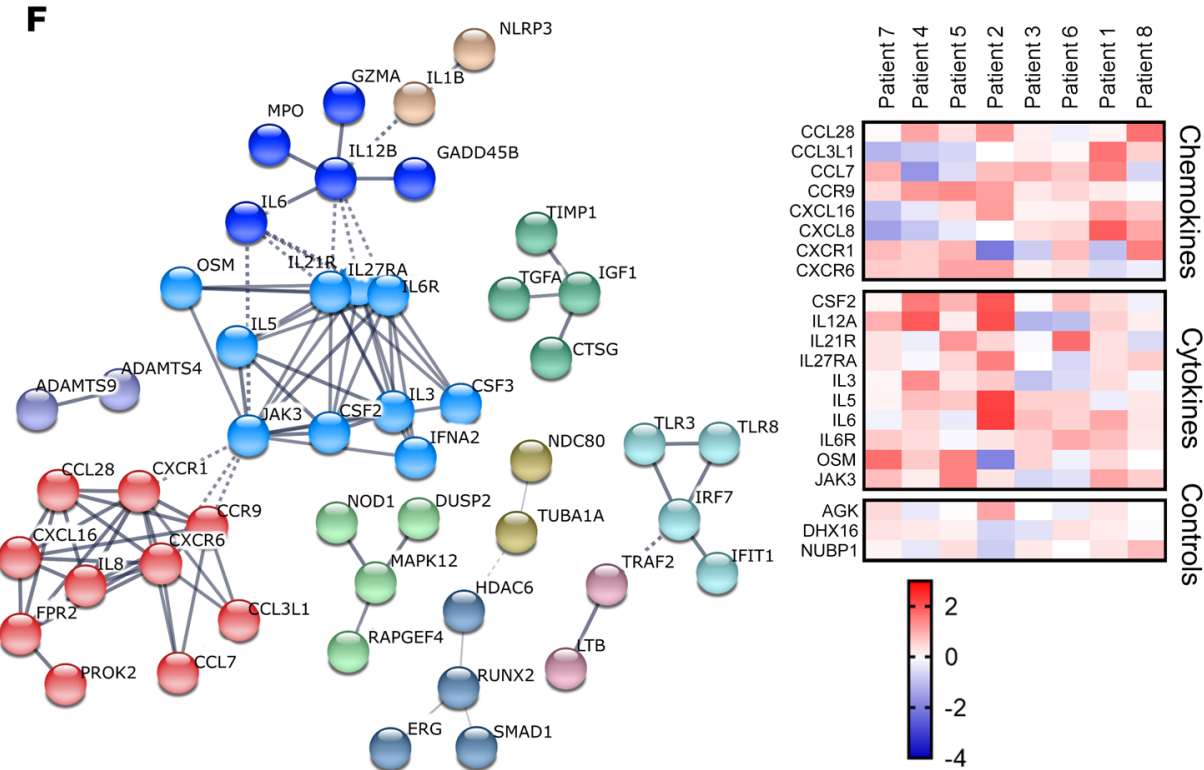
Figure 4. Anti-SIRP $\alpha \mathrm{mAb}$ reinvigorates myeloid cells in the human TME. (A) Fresh human surgical tumor explants (HCC, $n=7$; RCC, $n=2$; CRC, $n=1$; PDAC, $n=1$ ) dissociated in small organoids by enzymatic and mechanical digestion or cells from Meso $(n=7)$ were cultured for 48 hours with $10 \mu \mathrm{g} /$ $\mathrm{mL}$ of selective anti-SIRP $\alpha$ (OSE-172) or irrelevant control mAbs. Transcriptomic analysis was performed using the Human PCIP panel (NanoString). (B) nSolver relative gene expression analysis and volcano plot representation using the Benjamini-Hochberg method to adjust the $P$ value and the tumor origin as a confounding factor. (C) Heatmap representation of significantly overexpressed genes after treatment with anti-SIRP $\alpha$ (left). Gene expression signatures of main function identified in overexpressed genes as annotated by NanoString (right). (D) Myeloid cells from ovarian cancer ascites were isolated after Ficoll separation of ascites fluid and magnetic separation of $\mathrm{CD} 14^{+}$cells. Isolated myeloid cells were cultured for 48 hours with $10 \mu \mathrm{g} / \mathrm{mL}$ of selective anti-SIRP $\alpha$ (OSE-172) or irrelevant control mAbs. Transcriptomic analysis was performed using the Myeloid Innate Immunity panel_v2 (NanoString). (E) GSEA of functional gene expression signatures identified by $n$ Solver analysis as significantly overexpressed after anti-SIRP $\alpha$ treatment. (F) STRING protein-protein network analysis of the top 100 most overexpressed genes after anti-SIRP $\alpha$ treatment (left). Unsupervised heatmap representation of individual patient gene expression from the 2 main functional clusters (chemokine and cytokines) identified by STRING analysis (right). ${ }^{*} P<0.05$; ${ }^{* * *} P<0.0001$, paired Mann-Whitney $U$ test.

superior clinical efficacy, albeit with increased immune-related adverse events (63). The identification of alternative therapeutic approaches is therefore needed for extending the benefits of immunotherapy beyond inflamed tumors. Simultaneously activating adaptive and innate immune responses otherwise inhibited in the TME is likely to overcome this limitation. Our study uncovers the role of the SIRP $\alpha$ innate immune checkpoint in controlling macrophage chemokine secretion in the TME and hence in promoting $\mathrm{T}$ cell exclusion from the tumor nest. Moreover, we show that blocking SIRP $\alpha$ signaling synergizes with T cell ICB and $\mathrm{T}$ cell costimulatory agonists in vivo. Importantly, we reveal significant differences in the activity of mAbs targeting either CD47 or SIRPa in humans. Indeed, while selectively blocking SIRP $\alpha$ preserves and enhances $T$ cells responses, interfering with the SIRP $\gamma / C D 47$ axis inhibits human $\mathrm{T}$ cell activation, cytokine secretion, and $\mathrm{T}$ cell migration $(\mathrm{P}<0.05)$.

Preclinical research mainly using human tumor-xenograft models in immunodeficient mice shows that CD47 blockade effectively promotes tumor elimination by increasing macrophage phagocytosis (51-55). It was also shown that CD47 blockade synergizes with therapeutic antitumor antigen antibodies by enhancing antibody-dependent macrophage phagocytosis (42). A recent phase $1 \mathrm{~b}$ clinical trial on relapsed and refractory $\mathrm{B}$ cell non-Hodgkin's lymphoma patients showed that anti-CD47 mAbs have very promising activity when used in combination with the rituximab anti-CD20 mAb (64). However, due to the ubiquitous expression of CD47, including in erythrocytes and platelets, anti-CD47 therapeutic strategies have a limited therapeutic window, as the high dosages needed to overcome antigen sink entail safety risks for the patients. Moreover, while anti-CD47 mAbs increase macrophage phagocytosis of CD47-expressing tumor cells, they also affect other CD47-ligand pathways, and we currently have limited knowledge of the clinical consequences of interfering with these pathways. Although SIRPa is expressed by the central and peripheral nervous system $(65,66)$, no toxicity has been reported in
SIRP $\alpha$-deficient mice (67) and targeting CD47, which is ubiquitously expressed, hence also by the nervous system, was not associated with increased risk of neurological adverse events $(44,64,68,69)$. The preclinical efficacy of anti-SIRP $\alpha$ mAbs has been previously reported (57); however, the therapeutic potential of SIRP $\alpha$ antagonists in SIRP $\alpha$-negative tumors was not addressed. Indeed, monotherapy with anti-SIRP $\alpha$ mAbs (with Fc-effector ADCC and ADCP function) has been assessed in heterotopic models of melanoma and renal carcinoma, which are tumors that express unusually high levels of SIRP $\alpha$ (57). Moreover, in SIRP $\alpha$-negative tumor xenograft models, anti-SIRP $\alpha$ mAbs have only been tested in combination with cytotoxic antibodies (rituximab, cetuximab) that are known to induce antibody-dependent macrophage phagocytosis $(56,70)$.

To evaluate the clinical potential of anti-SIRP $\alpha$ mAbs and understand how SIRP $\alpha$ signals modify the TME, we tested noncytotoxic antagonist anti-SIRP $\alpha$ mAbs on SIRP $\alpha$-negative tumor syngeneic and orthotopic mouse models. We found that blocking SIRP $\alpha$ signaling was sufficient for modifying the TME. Moreover, anti-SIRP $\alpha$ monotherapy was effective in inhibiting tumor growth and preventing tumor metastasis in triple-negative breast cancer and mesothelioma orthotopic models that are highly enriched in myeloid cells. In models where anti-SIRP $\alpha$ monotherapy showed weak efficacy (e.g., orthotopic HCC or subcutaneous CRC models), a strong synergistic association was found with immunotherapies promoting $\mathrm{T}$ cell responses, such as $\mathrm{PD} 1 / \mathrm{PD}-\mathrm{L} 1$ antagonists or a $4-1 \mathrm{BB}$ costimulatory agonist. Most of the mice (60\%-80\%) treated with SIRP $\alpha / \mathrm{PD}-\mathrm{L} 1$ or SIRP $\alpha / 4-1 \mathrm{BB}$ combination therapy eradicated the primary tumor and developed robust and durable adaptive immune memory.

Transcriptomic and histological analyses revealed major TME modification upon SIRPa blockade, including accumulation of $\mathrm{T}$ lymphocytes within the tumor nest that were otherwise found restricted to the tumor margin. In parallel, significant overexpression of genes encoding for chemokines and cytokines was measured in the TME $(P<0.05)$. These results suggest that anti-SIRP $\alpha$ mAbs may reinvigorate chemokine secretion by myeloid cells in the TME. Indeed, ex vivo experiments using a human whole-tumor organoid model or purified $\mathrm{CD} 14^{+}$myeloid cells from ovarian carcinoma ascites showed that anti-hSIRP $\alpha$ mAbs induce significant upregulation of chemokine gene expression $(P<0.05)$. Finally, human macrophages cultured in vitro on CD47-coated plates or with different human tumor cell lines exhibited significant inhibition of chemokine secretion $(P<0.01)$, which was reversed by selective anti-hSIRP $\alpha \mathrm{mAb}$. Administration of the selective antihSIRP $\alpha \mathrm{mAb}$ in vivo in humanized mice promoted human $\mathrm{T}$ cell infiltration where human macrophages were implanted as well as in human tumor spheroids infiltrated by myeloid cells. Together, these results demonstrate that a high CD47-expression environment, such as a solid TME, may inhibit not only macrophage phagocytosis, but also chemokine secretion by myeloid cells via the SIRP $\alpha / C D 47$ axis, thereby contributing to $T$ cell exclusion from the tumor nest and resistance to anti-PD1/PD-L1 immunotherapies.

Anti-CD 47 mAbs promote antigen crosspresentation by DCs and increase antigen-specific $\mathrm{T}$ cell activation in mouse $(31,44)$. In addition, it was recently reported that anti-CD47 antibodymediated phagocytosis of cancer cells by macrophages can also generate cytotoxic $\mathrm{CD} 8^{+}$antitumor $\mathrm{T}$ cell responses in immuno- 


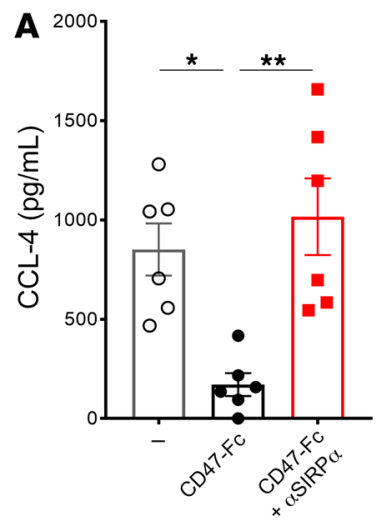

B

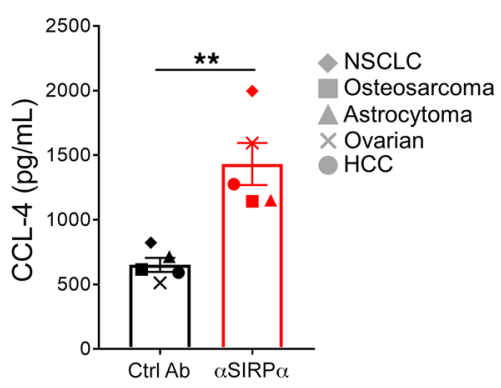

E
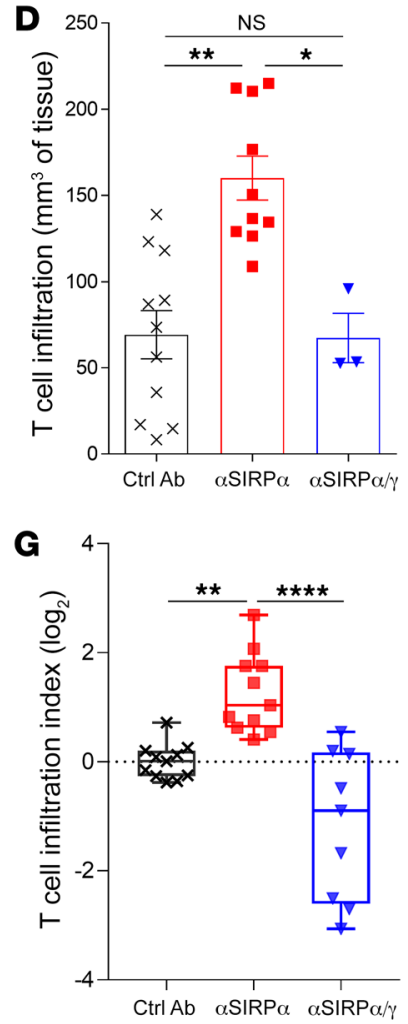

H

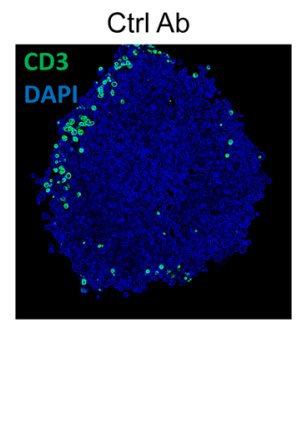

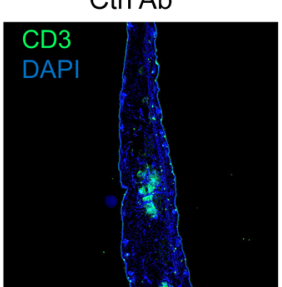

C

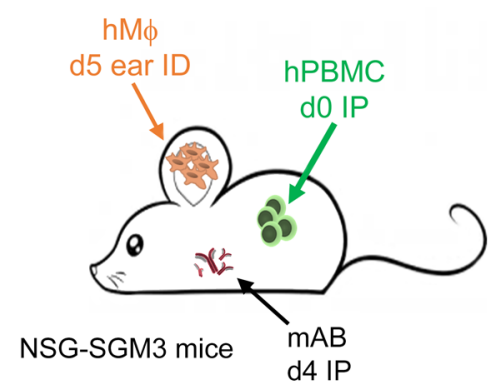

$\mathbf{F}$

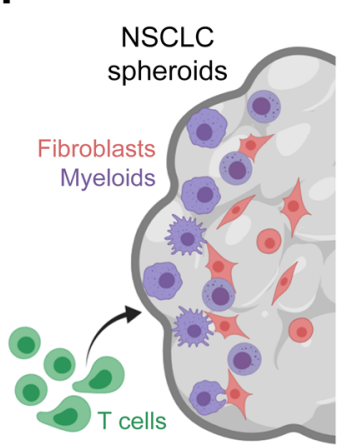

$\alpha \mathrm{SIRP} \alpha / \gamma \mathrm{mAB}$

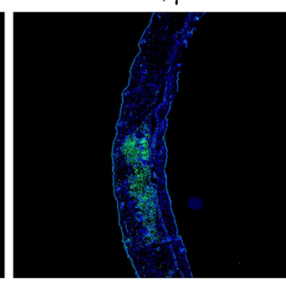

$\alpha \operatorname{SIRP} \alpha \operatorname{mAB}$

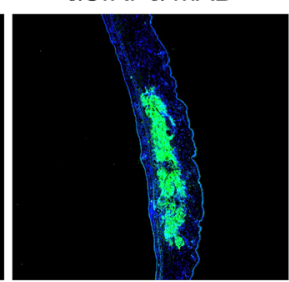

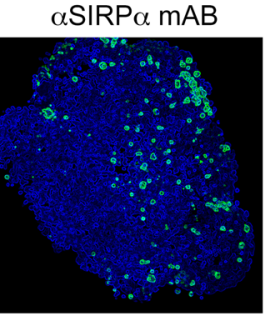

$\alpha \operatorname{SIRP} \alpha / \gamma \mathrm{mAB}$

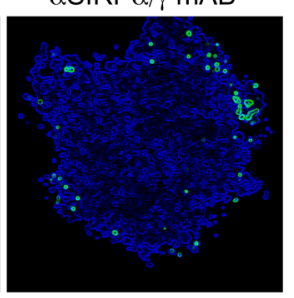

Figure 5. Anti-SIRPa mAb reinstates myeloid cell chemokine secretion and human T cell migration. (A) CCL4 $(n=6)$ chemokine secretion of human macrophages cultured for 24 hours on normal or CD47-coated plates with or without anti-SIRP $\alpha \mathrm{mAb}(\mathrm{OSE}-172,10 \mu \mathrm{g} / \mathrm{mL})$. (B) CCL4 secretion of human monocytes cultured 24 hours in contact with different human tumor cell lines and $10 \mu \mathrm{g} / \mathrm{mL}$ of anti-SIRP $\alpha \mathrm{mAb}(\mathrm{OSE}-172)$ or irrelevant control Ab (1 representative donor of $n=5$ ). (C) Immunodeficient NSG-SGM3 mice (expressing human IL3, GM-CSF and SCF) humanized with $30 \times 10^{6}$ human PBMCs injected i.p. were treated i.p. on day 4 with $10 \mathrm{mg} / \mathrm{kg}$ of selective anti-SIRP $\alpha \mathrm{mAb}$ (OSE-172, $n=10$ ), a nonselective anti-SIRP $\alpha / \gamma \mathrm{mAb}(\mathrm{Kwar} 23, n=3)$, or an irrelevant control mAb $(n=11)$. Intradermal injection in the ear of autologous human macrophages (generated in vitro from blood monocytes cultured 5 days with $100 \mathrm{ng} / \mathrm{mL}$ M-CSF) was performed on day 5. (D) The ear was collected for immunohistological analysis 6 hours after macrophage injection. T cell infiltration was quantified per $\mathrm{mm}^{3}$ of tissue using quantification of $\mathrm{CD3}^{+}$cells on serial sections from each individual mouse. ${ }^{*} P<0.05$; ${ }^{* *} P<0.01$, unpaired Mann-Whitney $U$ test. (E) Representative example of human CD3 staining (green) and nucleus (blue) from each group in $\mathbf{D}$. (F) A549 NSCLC cell line was cultured with human monocytes, MRC-5 fibroblasts, and indicated monoclonal antibodies at $10 \mu \mathrm{g} / \mathrm{mL}$. After 3 days, human T lymphocytes were added on established spheroids and cultured for an additional 3 days. (C) Spheroids were fixed on day 6 and stained with anti-human CD3 Ab; T cell invasive index was calculated according to frequency and distance from periphery of $\mathrm{CD}^{+}$cells. (H) Representative example of human $\mathrm{CD} 3$ staining (green) and nucleus (blue) from each group in G. Original magnification, $\times 100(\mathbf{E}) ; \times 200(\mathbf{H}) .{ }^{* *} P<0.01 ;{ }^{* * *} P<0.0001$, Kruskal-Wallis and Dunn's multiple comparisons tests.

competent mice (30). Consistent with these data, we found that anti-mSIRP $\alpha$ mAbs promoted antigen-specific $\mathrm{CD}^{+} \mathrm{T}$ cell proliferation by SIRP $\alpha^{+}$DCs in mouse. However, these mechanisms with anti-CD47 mAbs could not be translated to human models, likely due to the poorly understood role of the SIRP $\gamma$ homologue in human $\mathrm{T}$ cell biology. Preliminary reports indicate that SIRP $\gamma$ may be involved in $\mathrm{T}$ cell adhesion, costimulation, and transendothelial migration processes $(49,50)$. We found that a selective human SIRPa antagonist antibody (i.e., that does not bind to SIRP $\gamma$ ) enhances crosspriming by human DCs and antigen-specific human IFN- $\gamma \mathrm{T}$ cell response. In contrast, human $\mathrm{T}$ cell response remained unchanged in this crosspriming assay with anti-CD47 
mAbs or a nonselective anti-hSIRP $\alpha / \gamma \mathrm{mAb}$, contradicting our findings in mouse models. This discrepancy between the activity of anti-CD47 mAb in mice and humans might be explained by differences in DC biology or, alternatively, by a potential deleterious effect of these antibodies on human $\mathrm{T}$ cells. Indeed, experiments using chimeric mouse T cell clones lacking SIRP $\gamma$ expression and cultured with human DCs showed that anti-CD47 and anti-hSIRPa mAbs can trigger $\mathrm{T}$ cell responses. Consistent with this, polyclonal and allogeneic human $\mathrm{T}$ cell stimulation assays showed that anti-CD47 mAbs induced immunosuppressive effects, whereas the selective anti-hSIRP $\alpha$ mAb preserved T cell responses. Moreover, anti-CD47 mAbs also had a negative impact on human $\mathrm{T}$ cell transendothelial migration in vitro, in agreement with our in vivo data in humanized mice showing that selective anti-hSIRP $\alpha \mathrm{mAb}$ promoted human $\mathrm{T}$ cell migration in tissue infiltrated with human macrophages while the nonselective anti-human SIRP $\alpha / \gamma$ antagonist mAb did not. Together, these results reveal that the SIRP $\gamma /$ $\mathrm{CD} 47$ axis plays a previously underappreciated role in human $\mathrm{T}$ cell biology and suggest that, in humans, immunotherapies selectively targeting SIRP $\alpha$ differ mechanistically from those targeting CD 47.

This study shows that selectively blocking SIRP $\alpha$ on myeloid cells even without tumor cell opsonization promotes antitumor responses and positively modifies the TME. Notably, the SIRP $\alpha$ / CD47 axis inhibits not only macrophage phagocytosis, but also chemokine secretion of human myeloid cells, thereby inducing $\mathrm{T}$ cell exclusion from the tumor nest and contributing to resistance to anti-PD1/PD-L1 immunotherapies. Finally, we uncover a role for the human-specific SIRP $\gamma / C D 47$ axis in promoting human antitumor $\mathrm{T}$ cell responses. Thus, selective blockade of SIRP $\alpha$ signaling may open novel treatment avenues to reinstate efficient adaptive immune responses against tumor antigens while offering different therapeutic indices than current anti-CD47-based approaches (ClinicalTrials.gov NCT03990233, investigating the therapeutic potential of the humanized anti-SIRPa mAb [OSE-172] used in this study as a treatment in cancer-bearing patients).

\section{Methods}

\section{Mice and reagents}

C57BL/6 and BALB/c mice were purchased from Janvier Laboratory and were kept in the Nantes SFR Bonamy animal facility. Hepatoma Hepa1.6 (ATCC), mesothelioma AK7 (provided by CRCINA), colon carcinoma MC38 (ATCC), and mammary carcinoma 4T1 (provided by CRCINA) cells were cultured in RPMI (Life Technologies), 10\% FBS, glutamine, and antibiotics at $37^{\circ} \mathrm{C}$ and $5 \% \mathrm{CO}_{2}$. Cells were harvested and resuspended in PBS for animal inoculation. Expression of SIRP $\alpha$, CD47, and PD-L1 by all cell lines upon IFN- $\gamma$ stimulation $(70 \mathrm{ng} / \mathrm{mL})$ for 24 hours or at steady state was evaluated by flow cytometry (Supplemental Figure 3). Anti-SIRP $\alpha$ antibodies (P84 and MY-1 hybridomas) were provided by Takashi Matozaki, Institute for Academic Initiatives (IAI), Osaka University $(57,71)$ and produced from hybridoma by the Plateforme Protéines Recombinantes of the CRTI-UMR1064 laboratory. The MY-1 clone was recombinantly modified from a rat IgG2a to a mIgG1 antibody (MY1-mG1) to prevent ADCC and ADCP function of the IgG2a isotype. Anti-4-1BB mAb was produced from the $3 \mathrm{H} 3$ hybridoma (provided by R.S. Mittler, Department of Surgery, Emory University, Atlanta, Georgia, USA). Anti-mouse PD-L1 mAbs (clone 10F-9G2), anti-mouse CD47 mAbs (clones MIAP301 and MIAP410), and anti-human CD47 mAbs (clone B6H12- $\alpha$ CD47 no. 1) were purchased from Bio X Cell. Anti-human CD47 mAb no. 2 (clone CC2C6) and isotype control hIgG4 were purchased from BioLegend, and selective anti-SIRP $\alpha$ (clone OSE-172-hIgG4 format; engineered by OSE Immunotherapeutics) and nonselective anti-SIRP $\alpha / \gamma$ (clone Kwar23-hIgG4 format; from WO2015138600A2) were produced and purified by OSE Immunotherapeutics.

\section{Preclinical tumor models}

Murine triple-negative breast cancer $4 \mathrm{~T} 1$ cells $\left(0.25 \times 10^{6} /\right.$ mouse $)$ were injected into the fat of the mammary glands of 8 -week-old $\mathrm{BALB} / \mathrm{c}$ female mice. Tumor development was measured 3 times a week, and metastasis spreading was analyzed by macroscopic and microscopic evaluation in the lung after euthanasia on day 30. The HCC preclinical model was performed as previously described (72). Briefly, the hepatoma Hepa 1.6 cells $\left(2.5 \times 10^{6} /\right.$ mouse $)$ were injected through the portal vein of 8 -week-old C57BL/6J male mice. Tumor rechallenge was performed by injecting $2.5 \times 10^{6} \mathrm{Hepa} 1.6$ cells into the spleen. For adoptive transfer, cured mice and naive mice were sacrificed from 10 to 21 days after the new challenge with Hepa1.6 cells. Total splenocytes were collected and single-cell suspensions were prepared and injected intravenously into naive mice $(200 \mu \mathrm{L} /$ mouse) inoculated with Hepa1.6 cells on the same day. The mesothelioma AK7 cells $\left(3 \times 10^{6} /\right.$ mouse $)$ were injected into the pleural cavities of 8-week-old WT C57BL/6J female mice or in SIRP $\alpha$ mutant female mice (59). Memory response was analyzed by challenging cured mice with a new load of $3 \times 10^{6} \mathrm{AK} 7$ cells i.p.

The CRC MC38 cells $\left(0.5 \times 10^{6}\right.$ cells/mouse $)$ were injected subcutaneously into 8-week-old C57BL/6J male mice. The tumor development was measured 3 times a week and calculated as follows: ((length $\times$ width)exponent 1.5 ) $\times \pi / 6$ ).

\section{TIL immunophenotyping}

For both mammary carcinoma-infiltrating cells and liver nonparenchymal cell (NPC) preparation, tumors were digested with collagenase IV (MilliporeSigma) followed by Percoll (GE Healthcare) density-gradient centrifugation at $400 \mathrm{~g}$ only for liver tumors and RBC lysis. Then, TILs were stained with fluorochrome-conjugated anti-mouse antibodies: CD11b (clone M1/70), I/Ab (clone AF6-120.1) for HCC model or I/Ad (clone AMS-32.1) for 4T1 model, CD206 (clone MR5D3), CCR7 (clone 4B12), CD3e (clone 145-2C11), CD4 (clone RM4-5), CD8 (clone 53-6.7), CD25 (clone PC61), CD127 (clone A7R34), NK1.1 (clone PK136) for HCC model or NKp46 (clone 29A1.4) for 4T1 model, CD44 (clone IM7), CD62L (clone MEL-14), Ly6C (clone AL-21), Ly6G (clone 1A8), CD19 (clone 1D3), CD24 (clone M1/69), and CD38 (clone 90), all from BD Biosciences - Pharmingen. FACS analysis was conducted using a BD Biosciences - Pharmingen LSR-II, Canto-II flow cytometers, and FlowJo software (Tree Star).

\section{Human tumor organoids and ovarian cancer ascite cells models}

Human tumoral surgical pieces were digested with the Tumor Dissociation Kit (Miltenyi Biotec) with GentleMACS (Miltenyi Biotec) and split into a 48-well culture plate. Myeloid cells were isolated by magnetic positive selection of $\mathrm{CD}_{14}{ }^{+}$cells (CD14 microbeads; Miltenyi Biotec) from ascites of ovarian carcinoma patients collected by the CRB Biologie Cellulaire (CHU Pontchaillou-Rennes, Rennes, France) 
A

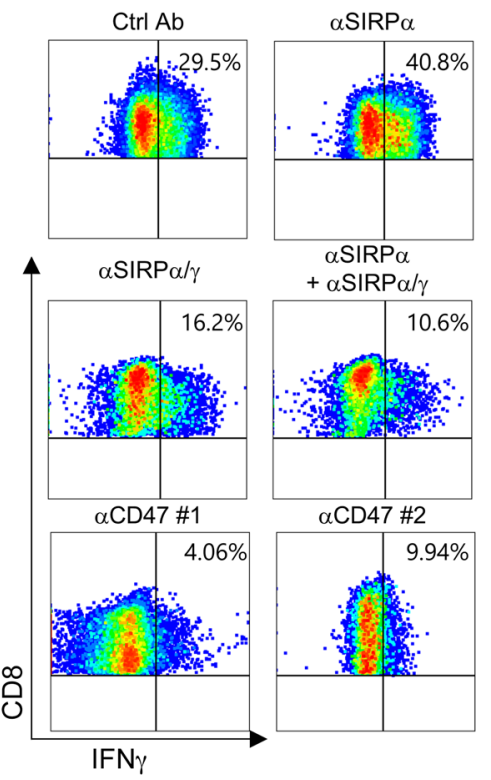

B

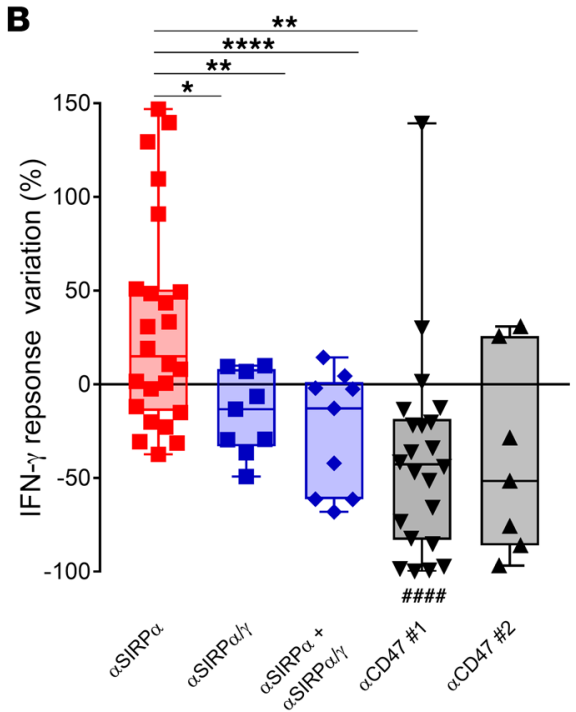

C

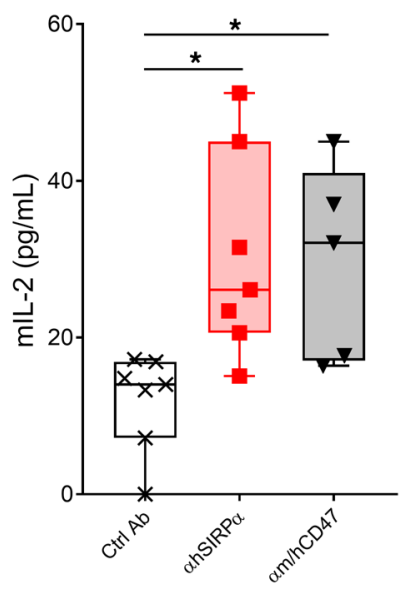

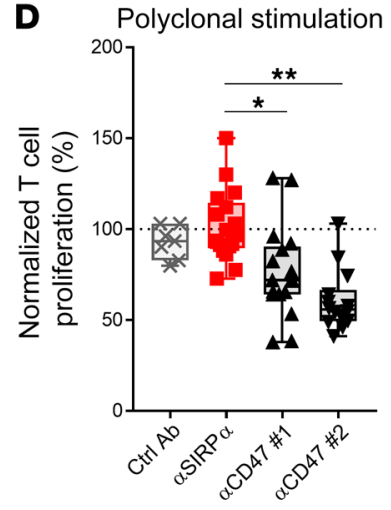

G

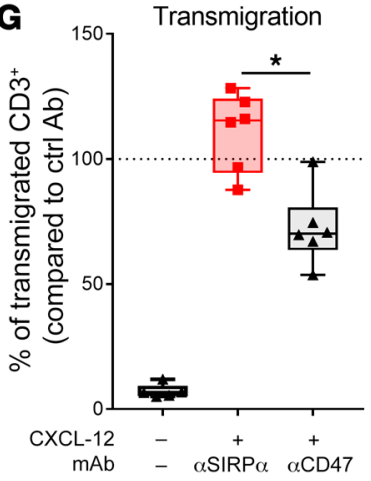

E Allogeneic stimulation

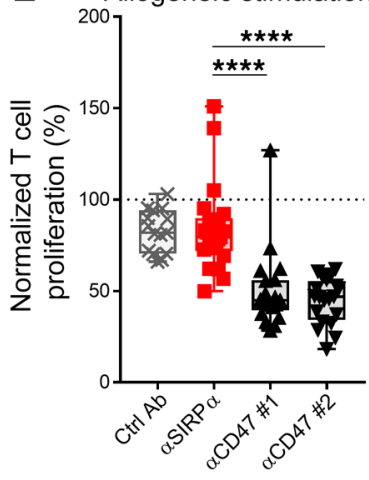

$\mathbf{F}$

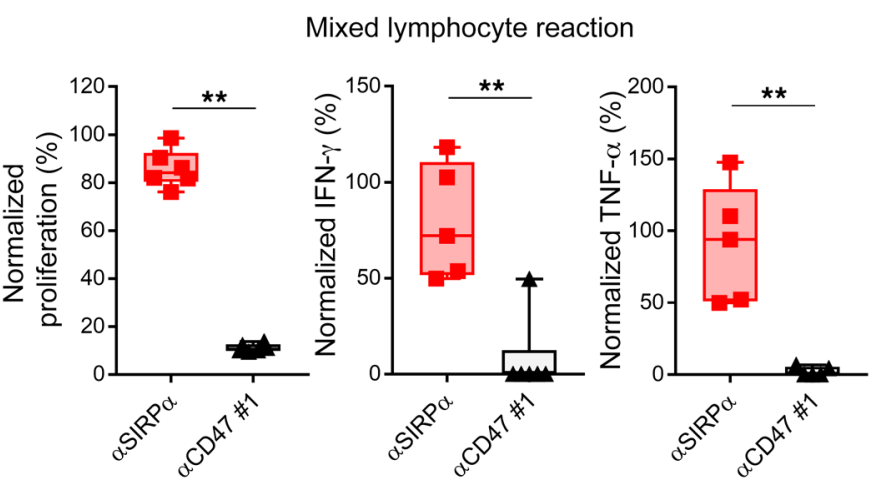

Figure 6. Selective targeting of SIRP $\alpha$ sparing SIRP $\gamma /$ CD47 preserves human T cell responses. (A) Representative IFN- $\gamma$ response of human HLA-A2/melan-A-specific CD8 ${ }^{+} T$ clones stimulated 6 hours with human HLA-A2 $D C s$ crosspresenting the long peptide melan-A/MART-1 and preincubated for 24 hours with the peptide and $10 \mu \mathrm{g} / \mathrm{mL}$ of different mAbs targeting the SIRP $\alpha /$ SIRP $\gamma /$ CD47 pathway. (B) Quantification of IFN- $\gamma$ response as in $\mathbf{A}$ normalized to the irrelevant control mAb condition for each DC donor. (C) IL-2 secretion by mouse chimeric thymoma cell line transfected with human CD8 and the HLA-A2/melan-A TCR cultured for 48 hours with human HLA-A2+ DC loaded with melan-A long peptide and cultured with indicated $\mathrm{mAbs}$, as in $\mathbf{A}$. (D) Proliferation of isolated human T cells from human PBMCs cultured with $10 \mu \mathrm{g} / \mathrm{mL}$ of indicated mAbs for 3 days with anti-CD3/anti-CD28-coated beads or (E) for 5 days with allogeneic LPS-matured human DCs. Proliferation measured by $\mathrm{H}^{3}$-thymidine incorporation was normalized under control conditions. (F) Human PBMCs from allogeneic healthy donors were cultured 5 days with indicated $\mathrm{mAbs}$ at $10 \mu \mathrm{g} / \mathrm{mL}$. T cell proliferation was assessed by $\mathrm{H}^{3}$-thymidine incorporation, and TNF- $\alpha$ and IFN- $\gamma$ secretion was quantified by ELISA and normalized to controls. (C) Human T cell migration across a monolayer of TNF- $\alpha$-activated endothelial cells with $10 \mu \mathrm{g} / \mathrm{mL}$ of indicated mAbs and CXCL-12 $(50 \mathrm{ng} / \mathrm{mL})$ as chemoattractant. The number of transmigrated $T$ cells was determined by flow cytometry after 4 hours and normalized under control conditions. ${ }^{*} P<$ 0.05 ; ${ }^{* *} P<0.01$; ${ }^{* * *} P<0.001$, between indicated conditions, unpaired Kruskall-Wallis test, Mann-Whitney $U$ test. $\# \# \# P<0.001$, compared with control Ab conditions.

and cultured in 96-well plates (50 000 cells/well). Antibodies were added at $10 \mu \mathrm{g} / \mathrm{mL}$, and cultures were performed for 48 hours.

\section{Transcriptomic analysis}

RNA from mouse and human tumoral tissues was extracted after a TRIzol-chloroform gradient and isolation with the RNeasy Mini Kit (QIAGEN). RNA from human myeloid cells isolated from ascites of ovarian carcinoma patients was extracted in the RLT buffer of the RNeasy Mini Kit (QIAGEN) supplemented by $\beta$-mercaptoethanol at
1\%. Gene expression was quantified with the NanoString nCounter platform using $50 \mathrm{ng}$ of total RNA for tumoral tissues or $25 \mathrm{ng}$ for isolated myeloid cells in either the nCounter Mouse PCIP Panel, the Human PCIP Panel, or the Human Myeloid Innate Immunity Panel, version 2 (NanoString Technologies). The code set was hybridized with the RNA overnight at $65^{\circ} \mathrm{C}$. RNA transcripts were immobilized and counted using the NanoString nCounter Sprint. Normalized expression data were analyzed with nSolver software. Genes extracted from heatmaps were tested for their protein interactions using 
STRING online software (https://string-db.org/). Gene set enrichment was evaluated using GSEA by phenotype permutation analysis based on 1000 permutations in cytokine and chemokine signatures designed by Nanostring.

\section{Immunofluorescence}

Liver samples from HCC tumor-bearing mice or MC38 colon carcinoma tumors and mouse ears from humanized NSG-SGM3 mice were frozen in Tissue-Tek (Thermo Fisher Scientific) and cut into $10 \mu \mathrm{m}$ sections. Immunofluorescence was performed with an antimouse CD3 Ab (clone 500A2), an anti-F4/80 Ab (clone CI:A3-1), or an anti-human CD3 Ab (catalog pAb A0452; Dako), and nucleus was revealed with DAPI. Sections were scanned and analyzed using standard fluorescence microscopy and NDPview2 imaging software (Hamamutsu). T cell and macrophage infiltration were analyzed by measuring the fluorescence intensity of the different channels related to the tumoral surface in a preclinical model of cancer. For the NSGSGM3 model, 5 serial sections were realized distanced by $100 \mu \mathrm{m}$ of thickness, and $\mathrm{T}$ cell infiltration was then related to a tumoral area.

\section{Chemokine secretion assay and in vivo humanized mice chemotaxis assay}

Blood from buffy coat of healthy volunteers was collected at the Etablissement Français du Sang (EFS), CHU Nantes-Hôtel Dieu (Nantes, France). hPBMCs were isolated after Ficoll density gradient centrifugation and RBC lysis. Monocytes were isolated by magnetic negative selection (Untouched Monocytes Isolation Kit II; Miltenyi Biotec) and cultured with either GM-CSF (10 ng/mL) for 2 days, then anti-SIRP $\alpha$ or anti-SIRP $\alpha / \gamma$ antibodies, and/or coated CD47-Fc protein $(10 \mu \mathrm{g} /$ $\mathrm{mL}$, Sino Biological) for 24 hours or different tumor cell lines HepG2 (HCC, ATCC), U2OS (osteosarcoma, ATCC), U373 (astrocytoma, ATCC), HeLa (ovarian carcinoma, ATCC), or A549 (NSCLC, ATCC) for 24 hours. CCL-3 and/or CCL-4 secretion in the supernatant was measured by ELISA (R\&D Systems). In vivo chemotaxis assay was performed in humanized NSG-SGM3 mice reconstituted with $30 \times 10^{6}$ i.p. injected hPBMCs. Anti-SIRP $\alpha$ (OSE-172), anti-SIRP $\alpha / \gamma$ (Kwar23), or isotype control (BioLegend) antibodies were administered i.p. at 10 $\mathrm{mg} / \mathrm{kg}$ at 4 days after reconstitution. Then mice received intradermal ear injection of autologous macrophages $\left(0.5 \times 10^{6}\right)$ differentiated from monocytes with M-CSF (R\&D Systems) at $100 \mathrm{ng} / \mathrm{mL}$ for 5 days. Ears were collected and frozen for histological analysis 6 hours after macrophage injection.

\section{Crosspresentation and T cell assays}

Murine crosspresentation assays. Splenic DCs were phenotyped using fluorochrome-conjugated anti-mouse antibodies from BD Biosciences - Pharmingen CD11c (clone HL3), CD11b (clone M1/70), CD8 $\alpha$ (clone 53-6.7), SIRPa (clone P84), H2Kd (clone AF6-88.5), CD86 (clone GL1), and CD103 (clone M290). Ex vivo crosspresentation was performed by sorting splenic DCs with a BD Biosciences ARIA-II flow cytometer according to their CD8 $\alpha$ and CD11b expression after CD11c enrichment by magnetic sorting (CD11c microbeads; Miltenyi Biotec) and by loading the cDC1 $\left(\mathrm{CD} 11 \mathrm{~b}^{-} \mathrm{CD} 8 \alpha^{+}\right)$or $\mathrm{cDC} 2\left(\mathrm{CD} 11 \mathrm{~b}^{+} \mathrm{CD} 8 \alpha^{-}\right)$ with $20 \mu \mathrm{g} / \mathrm{mL}$ of recombinant OVA overnight and with anti-SIRPa (P84 or MY1-mG1) or anti-CD47 (MIAP410) mAbs at $10 \mu \mathrm{g} / \mathrm{mL}$. Then OVA-loaded DCs were cultured with OT-I CD8 ${ }^{+}$cells isolated from OT-I transgenic mouse spleen by depleting CD8 ${ }^{-}$cells by magnetic sorting (CD8 Isolation Kit; Miltenyi Biotec) at a 1 DC:10 OT-I ratio for 72 hours. The proliferation was measured by $\mathrm{H}^{3}$-thymidine incorporation in the last 12 hours of culture. In vivo crosspresentation was performed by injecting i.p. MY1-mG1 or a control $\mathrm{Ab}$ at $10 \mathrm{mg} / \mathrm{kg}$ in naive mice 3 times at day -1 , day +1 , and day +3 , where do was the i.p. injection of 2.106 CPDe 450 (eBioscience) stained OT-I CD8 ${ }^{+} \mathrm{T}$ cells per mouse. Mice received $40 \mu \mathrm{g}$ of OVA i.p. at day +1 , and the spleen was collected at day +4 to assess OT-I proliferation and DC activation profile by flow cytometry. Cells were cultured in RPMI (Gibco; Thermo Fisher Scientific) supplemented by $10 \%$ FBS, glutamine, antibiotics, and $50 \mu \mathrm{M}$ of $\beta$-mercaptoethanol at $37^{\circ} \mathrm{C}$ and $5 \% \mathrm{CO}_{2}$.

Human crosspresentation assay and $\mathrm{T}$ cell stimulation. DCs were generated in vitro by differentiating HLA-A2 ${ }^{+}$monocytes isolated by negative magnetic selection (Untouched Monocytes Isolation Kit II; Miltenyi Biotech) with GM-CSF (50 ng/mL) and IL-4 (20 ng/mL) (both from CellGenix) for 6 days. Then DCs were loaded with a long peptide of melan-A/MART-1 (Proteogenix) for 24 hours with different Abs targeting the SIRP/CD47 pathway at $10 \mu \mathrm{g} / \mathrm{mL}$. HLA-A2/ melan-A-specific T cell clone (10C10) from N. Labarriere (Université de Nantes, CNRS, INSERM, CRCINA) (62) was cocultured for 6 hours with the melan-A-loaded DCs and stained for IFN- $\gamma$ secretion (B27) and CD8 expression (RPA-T8) by flow cytometry. The same Ab-treated melan-A-loaded DCs were cocultured with the SIRP $\gamma$-negative murine thymoma cell line, which is transgenic for human CD8 and melan-A/HLA-A2-specific TCR (provided in house), for 2 days, and IL-2 secretion was measured in the supernatant by ELISA according to the manufacturer's instructions (BD Biosciences). Polyclonal and allogeneic stimulations were performed on $\mathrm{T}$ cells isolated by negative magnetic selection (Pan T Cells; Miltenyi Biotec) from hPBMCs by 3 days of incubation with anti-CD3/anti-CD28-coated beads (Life Technologies) at a 1 bead to $2 \mathrm{~T}$ cells ratio or by 5 days of incubation with allogeneic induced DCs matured with $100 \mathrm{ng} / \mathrm{mL}$ of LPS (MilliporeSigma) at a $1 \mathrm{DC}$ to $5 \mathrm{~T}$ cells ratio, respectively, and incubated with $\mathrm{mAb}$ at $10 \mu \mathrm{g} / \mathrm{mL}$. Proliferation was measured by $\mathrm{H}^{3}$-thymidine incorporation during the last 18 hours of incubation. Mixed lymphocyte reaction was assessed by incubating fresh PBMCs with irradiated allogeneic PBMCs from different donors for 5 days with Abs at $10 \mu \mathrm{g} /$ $\mathrm{mL}, \mathrm{T}$ cell activation was assessed by thymidine incorporation as mentioned before, and TNF- $\alpha$ and IFN- $\gamma$ secretion were evaluated in the supernatant by ELISA (BD Biosciences). Cells were cultured in RPMI (Gibco; Thermo Fisher Scientific) supplemented by 10\% FBS, glutamine, and antibiotics at $37^{\circ} \mathrm{C}$ and $5 \% \mathrm{CO}_{2}$.

\section{Transendothelial migration assay}

Single donor primary human dermal microvascular endothelial cells (HDMEC) were purchased from Clonetics (Lonza) and cultured in complete EGM-2MV medium (Lonza). Cells were used between passages 5 to 8 and activated with TNF- $\alpha$ (R\&D Systems) at $100 \mathrm{U} /$ $\mathrm{mL}(2 \mathrm{ng} / \mathrm{mL})$ in the upper part of the 12-well Transwell overnight $\left(2 \times 10^{5} \mathrm{DMECs} /\right.$ well $)$. T cells were isolated by magnetic separation (Pan T Cell; Miltenyi Biotec), and $2 \times 10^{6} \mathrm{~T}$ cells were added in the upper part of the Transwell with Abs at $10 \mu \mathrm{g} / \mathrm{mL}$ after 15 minutes of preincubation of endothelial cells with CXCL-12 (50 ng/mL). Then, CXCL-12 was added at $50 \mathrm{ng} / \mathrm{mL}$ in the lower part of the Transwell and incubated for 4 hours at $37^{\circ} \mathrm{C}, 5 \% \mathrm{CO}_{2}$ in complete RPMI. After 4 hours of incubation, the number of transmigrated cells was determined by flow cytometry. 
For additional information, see Supplemental Methods.

\section{Statistics}

Continuous variables were expressed as mean \pm SEM, unless otherwise indicated, and raw data were compared with nonparametric tests, Mann-Whitney $U$ test for 2 groups or Kruskall-Wallis with Dunn's comparison when the number of groups was greater than 2 . The log-rank test was used to compare survival times between different groups. $P$ values of less than 0.05 were considered statistically significant. All statistical analyses were performed using GraphPad software.

\section{Study approval}

Animal housing and surgical procedures were conducted according to the guidelines of the French Agriculture Ministry and were approved by the Comité d'éthique en Expérimentation Animale-Pays de la Loire (Nantes, France) (Autorisation de Projet Utilisant des Animaux à des Fins Scientifiques [APAFIS] 646 and 9851). Human studies were performed with appropriate ethical approval from the Commission Nationale de l'Informatique et Libertés (CNIL) (Paris, France) and with informed written consent given by the patients.

\section{Author contributions}

$\mathrm{NP}$ and BV conceived the study. VG, BV, and NP designed and supervised the experiments. VG, SP, CM, EW, KLR, AM, JD, KB, MN, GP, VT, GT, CT, SD, PD, and BM performed experiments. VG, SP, CM, RA, AG, VV, RD, NV, SB, FH, CB, VC, PAG, CR, JFM, IA, EW, IG, KLR, EST, MM, SLBB, HA, SM, DC, NL, DL, SC, BV, and GB analyzed data and/or provided samples. VG, BV, and NP wrote the manuscript.

\section{Acknowledgments}

This work was supported by the French Public Bank of Investment (BPI EFFI-CLIN PSPC grant), the French Institut National du Cancer (INCA MDSCAN PRT-K15-136 grant), and the Regional Ligue Contre le Cancer 44. We thank the staff of the Humanized Rodent Platform and MicroPICell Cellular and Tissue Imaging Core Facility of Nantes University.

Address correspondence to: Nicolas Poirier, OSE Immunotherapeutics, 22 Boulevard Benoni Goullin, 44200 Nantes, France. Phone: 33.0.228.291.010; Email: nicolas.poirier@ose-immuno.com.
1. Sharma P, Allison JP. The future of immune checkpoint therapy. Science. 2015;348(6230):56-61.

2. Mellman I, Coukos G, Dranoff G. Cancer immunotherapy comes of age. Nature. 2011;480(7378):480-489.

3. Ribas A, Wolchok JD. Cancer immunotherapy using checkpoint blockade. Science. 2018;359(6382):1350-1355.

4. Galluzzi L, Chan TA, Kroemer G, Wolchok JD, López-Soto A. The hallmarks of successful anticancer immunotherapy. Sci Transl Med. 2018;10(459):eaat7807.

5. Joyce JA, Fearon DT. T cell exclusion, immune privilege, and the tumor microenvironment. Science. 2015;348(6230):74-80.

6. Jiang P, et al. Signatures of T cell dysfunction and exclusion predict cancer immunotherapy response. Nat Med. 2018;24(10):1550-1558.

7. Binnewies M, et al. Understanding the tumor immune microenvironment (TIME) for effective therapy. Nat Med. 2018;24(5):541-550.

8. Jenkins RW, Barbie DA, Flaherty KT. Mechanisms of resistance to immune checkpoint inhibitors. Br J Cancer. 2018;118(1):9-16.

9. Wei SC, Duffy CR, Allison JP. Fundamental mechanisms of immune checkpoint blockade therapy. Cancer Discov. 2018;8(9):1069-1086.

10. Cogdill AP, Andrews MC, Wargo JA. Hallmarks of response to immune checkpoint blockade. $\mathrm{Br} \mathrm{J}$ Cancer. 2017;117(1):1-7.

11. Sharma P, Hu-Lieskovan S, Wargo JA, Ribas A. Primary, adaptive, and acquired resistance to cancer immunotherapy. Cell. 2017;168(4):707-723.

12. Gentles AJ, et al. The prognostic landscape of genes and infiltrating immune cells across human cancers. Nat Med. 2015;21(8):938-945.

13. Mantovani A, Marchesi F, Malesci A, Laghi L, Allavena P. Tumour-associated macrophages as treatment targets in oncology. Nat Rev Clin Oncol. 2017;14(7):399-416.

14. Veglia F, Perego M, Gabrilovich D. Myeloidderived suppressor cells coming of age. Nat
Immunol. 2018;19(2):108-119.

15. De Henau O, et al. Overcoming resistance to checkpoint blockade therapy by targeting PI3K $\gamma$ in myeloid cells. Nature. 2016;539(7629):443-447.

16. Meyer C, et al. Frequencies of circulating MDSC correlate with clinical outcome of melanoma patients treated with ipilimumab. Cancer Immunol Immunother. 2014;63(3):247-257.

17. Highfill SL, et al. Disruption of CXCR2-mediated MDSC tumor trafficking enhances anti-PD1 efficacy. Sci Transl Med. 2014;6(237):237ra67.

18. Weber R, et al. Myeloid-derived suppressor cells hinder the anti-cancer activity of immune checkpoint inhibitors. Front Immunol. 2018;9:1310.

19. Bjoern J, et al. Immunological correlates of treatment and response in stage IV malignant melanoma patients treated with Ipilimumab. Oncoimmunology. 2016;5(4):e1100788.

20. Fridman WH, Zitvogel L, Sautès-Fridman C, Kroemer G. The immune contexture in cancer prognosis and treatment. Nat Rev Clin Oncol. 2017;14(12):717-734.

21. Mariathasan $S$, et al. TGF $\beta$ attenuates tumour response to $\mathrm{PD}-\mathrm{L} 1$ blockade by contributing to exclusion of T cells. Nature. 2018;554(7693):544-548.

22. Peranzoni E, et al. Macrophages impede CD8 T cells from reaching tumor cells and limit the efficacy of anti-PD-1 treatment. Proc Natl Acad Sci U S A. 2018;115(17):E4041-E4050.

23. Lesokhin AM, et al. Monocytic CCR2(+) myeloid-derived suppressor cells promote immune escape by limiting activated CD8 T-cell infiltration into the tumor microenvironment. Cancer Res. 2012;72(4):876-886

24. Zhu Y, et al. CSF1/CSF1R blockade reprograms tumor-infiltrating macrophages and improves response to T-cell checkpoint immunotherapy in pancreatic cancer models. Cancer Res. 2014;74(18):5057-5069.

25. Sánchez-Paulete AR, et al. Cancer immunotherapy with immunomodulatory anti-CD137 and anti-PD-1 monoclonal antibodies requires BATF3-dependent dendritic cells. Cancer Discov. 2016;6(1):71-79.

26. Salmon $\mathrm{H}$, et al. Expansion and activation of CD103(+) dendritic cell progenitors at the tumor site enhances tumor responses to therapeutic PD-L1 and BRAF inhibition. Immunity. 2016;44(4):924-938.

27. Spranger S, Dai D, Horton B, Gajewski TF. Tumor-residing Batf3 dendritic cells are required for effector $\mathrm{T}$ cell trafficking and adoptive $\mathrm{T}$ cell therapy. Cancer Cell. 2017;31(5):711-723.e4.

28. Simpson TR, et al. Fc-dependent depletion of tumor-infiltrating regulatory $\mathrm{T}$ cells co-defines the efficacy of anti-CTLA-4 therapy against melanoma. J Exp Med. 2013;210(9):1695-1710.

29. Willingham SB, et al. The CD47-signal regulatory protein alpha (SIRPa) interaction is a therapeutic target for human solid tumors. Proc Natl Acad Sci US A. 2012;109(17):6662-6667.

30. Tseng D, et al. Anti-CD47 antibody-mediated phagocytosis of cancer by macrophages primes an effective antitumor T-cell response. Proc Natl Acad Sci U S A. 2013;110(27):11103-11108.

31. Xu MM, et al. Dendritic cells but not macrophages sense tumor mitochondrial DNA for cross-priming through signal regulatory protein $\alpha$ signaling. Immunity. 2017;47(2):363-373.e5.

32. Matlung HL, Szilagyi K, Barclay NA, van den Berg TK. The CD47-SIRP $\alpha$ signaling axis as an innate immune checkpoint in cancer. Immunol Rev. 2017;276(1):145-164

33. Barclay AN, Van den Berg TK. The interaction between signal regulatory protein alpha (SIRP $\alpha)$ and CD47: structure, function, and therapeutic target. Annu Rev Immunol. 2014;32:25-50.

34. Sick E, Jeanne A, Schneider C, Dedieu S, Takeda K, Martiny L. CD47 update: a multifaceted actor in the tumour microenvironment of potential therapeutic interest. Br J Pharmacol. 2012;167(7):1415-1430.

35. Oldenborg PA, et al. Role of CD47 as a 
marker of self on red blood cells. Science. 2000;288(5473):2051-2054.

36. Zhao XW, et al. CD47-signal regulatory protein- $\alpha$ (SIRP $\alpha$ ) interactions form a barrier for antibody-mediated tumor cell destruction. Proc Natl Acad Sci U S A. 2011;108(45):18342-18347.

37. Majeti R, et al. CD47 is an adverse prognostic factor and therapeutic antibody target on human acute myeloid leukemia stem cells. Cell. 2009;138(2):286-299.

38. Uluçkan O, et al. CD47 regulates bone mass and tumor metastasis to bone. Cancer Res. 2009;69(7):3196-3204.

39. Chao MP, et al. Extranodal dissemination of non-Hodgkin lymphoma requires CD47 and is inhibited by anti-CD47 antibody therapy. Blood. 2011;118(18):4890-4901.

40. Edris B, et al. Antibody therapy targeting the CD47 protein is effective in a model of aggressive metastatic leiomyosarcoma. Proc Natl Acad Sci U S A. 2012;109(17):6656-6661.

41. Theocharides APA, et al. Disruption of SIRP $\alpha$ signaling in macrophages eliminates human acute myeloid leukemia stem cells in xenografts. JExp Med. 2012;209(10):1883-1899.

42. Weiskopf K, et al. Engineered SIRP $\alpha$ variants as immunotherapeutic adjuvants to anticancer antibodies. Science. 2013;341(6141):88-91.

43. Weiskopf K, et al. CD47-blocking immunotherapies stimulate macrophage-mediated destruction of small-cell lung cancer. JClin Invest. 2016;126(7):2610-2620.

44. Liu X, et al. CD47 blockade triggers T cellmediated destruction of immunogenic tumors. Nat Med. 2015;21(10):1209-1215.

45. Logtenberg MEW, et al. Glutaminyl cyclase is an enzymatic modifier of the CD47-SIRP $\alpha$ axis and a target for cancer immunotherapy. Nat Med. 2019;25(4):612-619.

46. Liu Q, et al. Inhibition of SIRP $\alpha$ in dendritic cells potentiates potent antitumor immunity. Oncoimmunology. 2016;5(9):e1183850.

47. Veillette A, Chen J. SIRP $\alpha$-CD47 immune checkpoint blockade in anticancer therapy. Trends Immunol. 2018;39(3):173-184.

48. Brooke G, Holbrook JD, Brown MH, Barclay AN. Human lymphocytes interact directly with CD47 through a novel member of the signal regulatory protein (SIRP) family. J Immunol. 2004;173(4):2562-2570.

49. Stefanidakis M, Newton G, Lee WY, Parkos CA, Luscinskas FW. Endothelial CD47 interaction with SIRPgamma is required for human T-cell transendothelial migration under shear flow conditions in vitro. Blood. 2008;112(4):1280-1289.

50. Piccio L, et al. Adhesion of human T cells to antigen-presenting cells through SIRPbeta2-CD47 interaction costimulates T-cell proliferation. Blood. 2005;105(6):2421-2427.

51. Gholamin S, et al. Disrupting the CD47-SIRPa anti-phagocytic axis by a humanized anti-CD47 antibody is an efficacious treatment for malignant pediatric brain tumors. Sci Transl Med. 2017;9(381):eaaf2968.

52. Zeng D, et al. A fully human anti-CD47 blocking antibody with therapeutic potential for cancer. Oncotarget. 2016;7(50):83040-83050.

53. Liu J, et al. Pre-clinical development of a humanized anti-CD47 antibody with anti-cancer therapeutic potential. PLoS One. 2015;10(9):e0137345

54. Petrova PS, et al. TTI-621 (SIRP $\alpha F c): A C D 47-b l o c k-$ ing innate immune checkpoint inhibitor with broad antitumor activity and minimal erythrocyte binding. Clin Cancer Res. 2017;23(4):1068-1079.

55. Piccione EC, et al. SIRP $\alpha$-antibody fusion proteins selectively bind and eliminate dual antigen-expressing tumor cells. Clin Cancer Res. 2016;22(20):5109-5119.

56. Ring NG, et al. Anti-SIRP $\alpha$ antibody immunotherapy enhances neutrophil and macrophage antitumor activity. Proc Natl Acad Sci U S A. 2017;114(49):E10578-E10585.

57. Yanagita T, et al. Anti-SIRP $\alpha$ antibodies as a potential new tool for cancer immunotherapy. JCI Insight. 2017;2(1):e89140.

58. Verjan Garcia N, et al. SIRP $\alpha / C D 172 a$ regulates eosinophil homeostasis. JImmunol. 2011;187(5):2268-2277.

59. Inagaki K, et al. SHPS-1 regulates integrin-mediated cytoskeletal reorganization and cell motility. ЕМВО J. 2000;19(24):6721-6731.

60. Zboralski D, Hoehlig K, Eulberg D, Frömming A, Vater A. Increasing tumor-infiltrating T cells through inhibition of CXCL12 with NOX-A12 synergizes with PD-1 blockade. Cancer Immunol Res. 2017;5(11):950-956.
61. Herter S, et al. A novel three-dimensional heterotypic spheroid model for the assessment of the activity of cancer immunotherapy agents. Cancer Immunol Immunother. 2017;66(1):129-140.

62. Vignard V, et al. Adoptive transfer of tumor-reactive Melan-A-specific CTL clones in melanoma patients is followed by increased frequencies of additional Melan-A-specific T cells. J Immunol. 2005;175(7):4797-4805.

63. Postow MA, Sidlow R, Hellmann MD. Immune-related adverse events associated with immune checkpoint blockade. N EnglJ Med. 2018;378(2):158-168.

64. Advani R, et al. CD47 Blockade by Hu5F9-G4 and rituximab in non-Hodgkin's lymphoma. N Engl JMed. 2018;379(18):1711-1721.

65. van den Nieuwenhof IM, Renardel de Lavalette C, Diaz N, van Die I, van den Berg TK. Differential galactosylation of neuronal and haematopoietic signal regulatory protein-alpha determines its cellular binding-specificity. J Cell Sci. 2001;114(Pt 7):1321-1329.

66. Adams S, et al. Signal-regulatory protein is selectively expressed by myeloid and neuronal cells. JImmunol. 1998;161(4):1853-1859.

67. Murata Y, Saito Y, Kotani T, Matozaki T. CD47-signal regulatory protein $\alpha$ signaling system and its application to cancer immunotherapy. Cancer Sci. 2018;109(8):2349-2357.

68. Sikic BI, et al. First-in-human, first-in-class phase I trial of the anti-CD47 antibody Hu5F9-G4 in patients with advanced cancers. J Clin Oncol. 2016;34(15_suppl):3019-3953.

69. Advani RH, et al. Activity and tolerabilty of the first-in-class anti-CD47 antibody Hu5F9-G4 with rituximab tolerated in relapsed/refractory non-Hodgkin lymphoma: Initial phase $1 \mathrm{~b} / 2$ results. JClin Oncol. 2018;36(15_suppl):7504.

70. Murata Y, et al. Anti-human SIRP $\alpha$ antibody is a new tool for cancer immunotherapy. Cancer Sci. 2018;109(5):1300-1308.

71. Chuang W, Lagenaur CF. Central nervous system antigen P84 can serve as a substrate for neurite outgrowth. Dev Biol. 1990;137(2):219-232.

72. Gauttier V, Judor JP, Le Guen V, Cany J, Ferry N, Conchon S. Agonistic anti-CD137 antibody treatment leads to antitumor response in mice with liver cancer. Int J Cancer. 2014;135(12):2857-2867. 